\title{
HAAR MEASURE FOR MEASURE GROUPOIDS ${ }^{1}$
}

\author{
BY
}

PETER HAHN

\begin{abstract}
It is proved that Mackey's measure groupoids possess an analogue of Haar measure for locally compact groups; and many properties of the group Haar measure generalize. Existence of Haar measure for groupoids permits solution of a question raised by Ramsay. Ergodic groupoids with finite Haar measure are characterized.
\end{abstract}

Introduction. In their pioneering investigation of operator algebras on Hilbert space, Murray and von Neumann [12] obtained examples of non-type I factors using ergodic actions of a group on a measure space. Since then their method has been adapted and generalized several times to yield interesting new examples of von Neumann algebras; but despite recasting, the procedure has always seemed special and somewhat mystifying.

The present work is the first of several papers treating Haar measure and convolution algebras of functions on George Mackey's measure groupoids [10]. This research permits us to interpret the construction made by Murray and von Neumann, as well as subsequent generalizations, in terms of these convolution algebras. Our unified treatment includes Krieger's construction of factors from nonfree actions of countable groups [7], Dixmier's examples of quasi-unitary algebras [2], and the regular representation of second countable locally compact groups. They all arise from modular Hilbert algebras of functions on some appropriate groupoid. Our approach seems natural because each step can easily be related to the special case of groups, which is widely known.

Consider, for example, the case of a group action. Let $g$ be a locally compact second countable group with Haar measure $h$; and suppose $(S, \mu)$ is a standard finite measure space on which $g$ acts so that $\mu$ remains invariant. We denote by $s x$ the transform of $s \in S$ by $x \in g$ and assume that $(s, x) \mapsto$ $s x: S \times \mathfrak{g} \rightarrow S$ is Borel measurable. The measure space $(S \times \mathfrak{g}, \mu \times h)$ becomes a measure groupoid with unit (or object) space $S$ if we define the product $(s, x)(t, y)=(s, x y)$ whenever $t=s x$. If $f$ and $g$ are suitably restricted functions on $S \times \mathrm{g}$, Dixmier [2] and Glimm [4] have used a product

Received by the editors May 5, 1976 and, in revised form, April 13, 1977.

AMS (MOS) subject classifications (1970). Primary 28A65, 28A70; Secondary 28-02.

${ }^{1}$ Preparation supported by NSF Grant MPS 74-19876

- American Mathematical Society 1978 


$$
f * g(s, x)=\int f(s, y) g\left(s y, y^{-1} x\right) d h(y),
$$

which reduces when $S$ is a single point to the usual convolution for locally compact groups. When $g$ and $S$ are chosen properly, the Murray and von Neumann examples are obtained as the von Neumann algebras generated by convolution on the left or on the right on the Hilbert space $L^{2}(S \times \mathfrak{g}, \mu \times h)$. Invariance of $\mu$ was assumed here only for convenience.

Although he was motivated to study measure-theoretic groupoids by consideration of the group action case just described, Mackey found that a thorough treatment required the study of more general groupoids which were not necessarily described by group actions. He introduced these more general groupoids in [10]. Mackey's groupoids are endowed by definition with a family of measures satisfying a kind of quasi-invariance condition. These measures are the generalization of the measures obtained in the group action case by the product of the measure $\mu$ on $S$ with measures in the Haar measure class on $\mathrm{g}$. Initial attempts to define a convolution in the general case failed because there was no obvious analogue of the measure $\mu \times h$ with its special invariance properties; this invariance permits Dixmier's and Glimm's product to be associative.

The main result of this paper is that the measure class of a general measure groupoid contains a $\sigma$-finite measure which is translation-invariant in the groupoid sense (Theorem 3.9). Thus we will have proved the existence of invariant measures in the sense discussed by Mackey in $\$ 4$ of [10]. This Haar measure for groupoids shares many of the properties of the Haar measure for groups, to which it reduces if the groupoid is a group. These properties will be exploited in a subsequent paper [6], where the Haar measure on the groupoid is used to define a satisfactory convolution for functions on the groupoid. The convolution, together with the modular function associated with the Haar measure, permits us to define modular Hilbert algebras for the groupoid. In the present paper, a fragment of the convolution theory is employed in the proof of Theorem 3.8, in order to show that the modular function is a homomorphism in the groupoid sense.

The existence of a Haar measure for groupoids answers a seemingly unrelated question posed by Ramsay [14]. A structure theorem for the Haar measure (Theorem 4.4) is derived for this purpose. The final topic discussed in this paper is the case of an ergodic groupoid with finite Haar measure. The characterization we give is satisfactory: such groupoids define the same virtual group in the sense of Mackey [11] as a certain compact group.

The research reported here comprises part of the author's doctoral thesis [5]. The author wishes to thank the National Science Foundation for providing him, as one of its Graduate Fellows, with support during his first three 
graduate years. He is deeply grateful for the astute direction provided by his thesis adviser, Professor Mackey.

1. Algebraic groupoids. In this section we provide a minimum of background about groupoids outside of the measure-theoretic setting. A more leisurely exposition is found in [13, §1].

Definition 1.1. A groupoid is a set $G$, together with a distinguished subset $G^{(2)} \subset G \times G$, and maps $(x, y) \mapsto x y: G^{(2)} \rightarrow G$ (product) and $x \mapsto x^{-1}$ : $G \rightarrow G$ (inverse) such that

1. $\left(x^{-1}\right)^{-1}=x$.

2. $(x, y) \in G^{(2)}$ and $(y, z) \in G^{(2)} \Rightarrow(x y, z) \in G^{(2)}$ and $(x, y z) \in G^{(2)}$, and $(x y) z=x(y z)$.

3. $\left(x^{-1}, x\right) \in G^{(2)}$; and if $(x, y) \in G^{(2)}$, then $x^{-1}(x y)=y$.

4. $\left(x, x^{-1}\right) \in G^{(2)}$; and if $(z, x) \in G^{(2)}$, then $(z x) x^{-1}=z$.

Thus, a groupoid is a small category with inverses. Defining $r(x)=x x^{-1}$ (the range map) and $d(x)=x^{-1} x$ (the domain map), the objects or units of this category may be identified with $U_{G}=r(G) .(x, y) \in G^{(2)}$ iff $d(x)=$ $r(y)$; and $r(x)=x$ iff $x \in U_{G}$ iff $d(x)=x$. The cancellation laws hold; e.g. $x y=x z$ iff $y=z$.

The definition we have given was suggested by Mackey in a conversation. This definition can be verified to be equivalent to the one given on p. 255 of [13].

$G$ is a group iff $G^{(2)}=G \times G$ iff $U_{G}$ consists of exactly one element. We define for $E \subset G, E^{-1}=\left\{y \in G: y^{-1} \in E\right\}, x E=\left\{y \in r^{-1}(r(\{x\})): x^{-1} y\right.$ $\in E\}$, and $E x=\left(x^{-1} E^{-1}\right)^{-1}$.

EXAMPLE 1.2. Let $S$ be a set, $g$ a group acting on $S$ such that for $s \in S$ and $x \in \mathrm{g}, s x$ denotes the transform of $s$ by $x$. Let $G=S \times \mathfrak{g}, G^{(2)}=\{((s, x)$, $(t, y)) \in(S \times g) \times(S \times g): t=s x\}$. Define the product $(s, x)(s x, y)=$ $(s, x y)$ and inverse $(s, x)^{-1}=\left(s x, x^{-1}\right)$. Then $G$ is a groupoid with units $U_{G}=S \times\{e\}$. In subsequent consideration of this example, we will identify $S$ with $U_{G}$.

EXAMPLE 1.3. If $G$ is a groupoid, $G^{(2)}$ can also be made a groupoid. $G^{(2)(2)}=\left\{((x, y),(z, w)) \in G^{(2)} \times G^{(2)}: z=x y\right\}$. The multiplication is $(x, y)(x y, w)=(x, y w)$ and inversion is $(x, y)^{-1}=\left(x y, y^{-1}\right)$. Since $r^{(2)}(x, y)$ $=(x, y)\left(x y, y^{-1}\right)=(x, r(y))=(x, d(x))$ and $d^{(2)}(x, y)=(x y, d(x y))$, the unit space may be identified with $G$.

EXAMPLE 1.4. Let $G$ be a groupoid, $E$ a subset of $U_{G}$. Let $G \mid E=\{x \in G$ : $r(x) \in E$ and $d(x) \in E\} . G \mid E$ becomes a groupoid with units $E$ if we define $(G \mid E)^{(2)}=G^{(2)} \cap(G|E \times G| E)$.

Definition 1.5. The groupoid $G \mid E$ is called the reduction of $G$ by $E$.

The word contraction has also been used. 
Definition 1.6. A groupoid $G$ is called principal if $x \mapsto(r(x), d(x))$ : $G \rightarrow U_{G} \times U_{G}$ is one-one.

EXAMPLE 1.7. In Example 1.2, suppose $s x=s \Rightarrow x=e$. The action of $g$ on $S$ is called free. If $s x=s y$, then $x=y$; hence $(s, x)$ is determined by $s$ and $s x$. Consequently, the groupoid $S \times \mathrm{g}$ is principal.

EXAMPLE 1.8. Let $\delta$ be an equivalence relation on the set $S$. Let $\mathcal{E}^{(2)}=$ $\{((s, t),(u, v)) \in \mathcal{E} \times \mathcal{E}: t=u\}$. With product $(s, t)(t, v)=(s, v)$ and inverse $(s, t)^{-1}=(t, s), \mathcal{E}$ is a principal groupoid such that $U_{\mathscr{E}}$ may be identified with $S . r(s, t)=s$ and $d(s, t)=t . G^{(2)}$ in Example 1.3 is isomorphic to the equivalence relation established on $G$ by the right multiplication action (see $\S 1$ of [13]) of $G$ on itself.

If $G$ is any groupoid, then $\left\{(u, v) \in U_{G} \times U_{G}: \exists x \in G\right.$ such that $r(x)=u$ and $d(x)=v\}$ is an equivalence relation on $U_{G}$. It is the image of the map $x \mapsto(r(x), d(x))$. If $u, v \in U_{G}$ we write $u \sim v$ if $u$ and $v$ are equivalent with respect to this relation. The groupoid defined by this equivalence relation as in Example 1.8 is called the principal groupoid associated with $G$ and denoted $(r, d)(G)$. Its unit set is identified with $U_{G}$.

Definition 1.9. Let $G$ and $H$ be groupoids. A function $\rho: G \rightarrow H$ is a homomorphism if $(\rho(x), \rho(y)) \in H^{(2)}$ and $\rho(x) \rho(y)=\rho(x y)$ whenever $(x, y) \in$ $G^{(2)}$. We denote by $\tilde{\rho}$ the restriction of $\rho$ to $U_{G}$.

Note that since $\rho\left(x^{-1}\right) \rho(x) \rho(y)=\rho\left(x^{-1} x y\right)=\rho(y), \rho\left(x^{-1}\right)=\rho(x)^{-1}$. Hence $\tilde{\rho}\left(x x^{-1}\right)=\rho\left(x x^{-1}\right)=\rho(x) \rho\left(x^{-1}\right) \in U_{H}$.

EXAMPLE 1.10. The map $x \rightarrow(r(x), d(x))$ is a homomorphism of $G$ onto its associated principal groupoid.

EXAmPLE 1.11. For groupoids $G=S \times g$ of Example 1.2, a homomorphism of $G$ into a group $H$ is a function $\rho$ such that $\rho(s, x y)=$ $\rho(s, x) \rho(s x, y)$, a one-cocycle.

2. Measure groupoids. Before giving the definition, we will state some conventions and facts about measure theory. See [8], [9], [13].

By a Borel space we mean a set $S$, together with a $\sigma$-algebra $\mathscr{B}(S)$ of subsets of $S$, called Borel sets. $(S, \Re(S))$ is called countably separated if there is a sequence $\left(E_{i}\right)$ of Borel sets separating the points of $S$; i.e., for every pair of distinct points of $S \exists i \in \mathbf{N}$ such that $E_{i}$ contains one point but not both. A function from one Borel space into another is itself called Borel if the inverse image of every Borel set is Borel. A one-one onto function Borel in both directions is called a Borel isomorphism.

The Borel sets of a complete separable metric space are taken to be the $\sigma$-algebra generated by the open sets. The Borel sets of any subset of a Borel space are taken to be the relative Borel sets. $(S, \mathscr{B}(S))$ is called standard if it is Borel isomorphic to a Borel subset of a complete separable metric space. There is up to Borel isomorphism exactly one uncountable standard space. A 
countably separated space which is the image of a Borel function from a standard space is called analytic.

By a measure on $(S, \mathscr{B}(S)$ ) we always mean a countably additive $\sigma$-finite positive measure defined for elements of $\Re(S)$. By a probability we mean a measure with value 1 at $S \in \mathscr{B}(S)$. $\delta_{s}$ denotes the probability such that $\delta_{s}(E)=1$ if $s \in E, \delta_{s}(E)=0$ if $s \notin E$. Often we refer to $(S, \mu)$ as a measure space, or probability space if $\mu(S)=1$, without mentioning explicitly the Borel sets. A subset of $S$ or function on $S$ is called $\mu$-measurable if it is measurable with respect to the completion of $\mu$, which is again denoted $\mu$. The complement of a null set is called conull.

We write $\mu \ll \lambda$ if $\lambda(E)=0 \Rightarrow \mu(E)=0$ and say $\mu$ is equivalent to $\lambda$ $(\mu \sim \lambda)$ if both $\mu \ll \lambda$ and $\lambda \ll \mu$. The measure class $[\mu]$ of $\mu \neq 0$ is the set of measures equivalent to $\mu$. Every measure class contains a probability. If $E$ is a set, $1_{E}$ is the characteristic function of $E$.

Analytic spaces are metrically standard; this means that if $(S, \mu)$ is an analytic measure space, there is a conull Borel subset $S_{0}$ of $S$ which is a standard space in its relative Borel structure. If $E \subset S$ is null, $E$ is a subset of a null Borel set. $\mathscr{B}(S)$ is generated by a countable subset of $\mathscr{B}(S)$. Analytic subsets of a countably separated space are universally measurable.

The following theorem is needed in order to define measure groupoid. It is derived from Lemma 4.4 of [3] and the facts already stated.

TheOREM 2.1. Let $(S, \lambda)$ be an analytic probability space, $T$ another analytic space, and $p: S \rightarrow T$ a Borel surjection. Suppose $\nu \sim \lambda$. Let $\tilde{\lambda}=p_{*} \lambda=(E \mapsto$ $\left.\lambda\left(p^{-1}(E)\right)\right)$. Let $P$ be a positive Borel function such that $P=d \nu / d \lambda$. There exists a function $t \mapsto \nu_{t}$ from $T$ into the set of measures on $S$ such that

1. If $f \geqslant 0$ is Borel on $S$ then $t \mapsto \int f d \nu_{t}$ is an extended real-valued Borel function.

2. $\nu_{t}\left(S-p^{-1}(\{t\})\right)=0$ for all $t \in T$.

3. If $f \geqslant 0$ is Borel on $S$ then $\int f d \nu=\int\left(\int f d \nu_{t}\right) d \tilde{\lambda}(t)$.

$t \mapsto \nu_{t}$ is determined by 1, 2, 3 up to modification on a $\tilde{\lambda}$-null Borel set. $t \mapsto \lambda_{t}$ is determined a.e. by properties 1 and 3 together with

$2^{\prime} . \tilde{\lambda}(E)=\iint 1_{E}{ }^{\circ} p d \lambda_{t} d \tilde{\lambda}(t)$ for $E$ in a generating subalgebra of $\mathscr{B}(T)$. Almost all $\lambda_{t}$ are probability measures and $P=d \nu_{t} / d \lambda_{t}$ a.e.

We say that $\lambda=\int \lambda_{t} d \tilde{\lambda}(t)$ is a $p$-decomposition of $\lambda$ and that $\nu=\int \nu_{t} d \tilde{\lambda}(t)$ is a $p$-decomposition of $\nu$ with respect to $\tilde{\lambda}$. The theorem implies that the measure classes $\left[\nu_{t}\right]$ are determined by $[\nu]$ up to a $[\tilde{\lambda}]$-null set. $[\nu]$ also determines $[\tilde{\lambda}]$.

Now we are prepared to give the definition of measure groupoid and some examples. We follow Mackey [10], [11] and Ramsay [13].

Let $G$ be a groupoid such that the underlying space is also endowed with a 
Borel structure. If $G^{(2)}$ is a Borel set in the product structure on $G \times G$, and $(x, y) \mapsto x y: G^{(2)} \rightarrow G$ and $x \mapsto x^{-1}: G \rightarrow G$ are Borel functions, then $G$ is called a Borel groupoid. $G$ is an analytic groupoid if the Borel si-ucture is analytic. Note that $r=x \mapsto x x^{-1}$ and $d=x \mapsto x^{-1} x$ are Borel anc $U_{G}=$ $r(G) \subset G$ is analytic if $G$ is.

Suppose that $G$ is an analytic groupoid and $\mu$ a probability on $r^{-1}(\{u\})$, where $u$ is a fixed unit of $G$. Then if $d(x)=u, E \mapsto \int 1_{E}(x y) d \mu(y)$ defines a probability $x \cdot \mu$ on $r^{-1}(\{r(x)\})$. The product $x y$ is defined for $\mu$-a.a. $y$ precisely because $\mu$ is supported by $r^{-1}(\{d(x)\})$.

If $\lambda$ is a measure on $G, \lambda^{-1}$ is defined by $\lambda^{-1}(E)=\dot{\lambda}\left(E^{-1}\right)$.

Definition 2.2. A measure $\lambda$ on $G$ is symmetric if $\lambda^{-1}=\lambda$. A measure class $C$ is called symmetric if a symmetric measure belongs to $C$.

It is immediate that if $\nu \sim \nu^{-1}$ then a symmetric probability measure belongs to $[\nu]$.

Definition 2.3. Let $C$ be a symmetric measure class on the analytic groupoid $G$. Let $\lambda \in C$ be a probability measure with $r$-decomposition $\lambda=\int \lambda^{u} d \tilde{\lambda}(u)$ over $U_{G} . \lambda$ is called (left) quasi-invariant if there is a $\tilde{\lambda}$-conull Borel set $U_{1} \subset U_{G}$ such that if $r(x) \in U_{1}$ and $d(x) \in U_{1}$, then $x \cdot \lambda^{d(x)} \sim$ $\lambda^{r(x)}$. A symmetric measure class $C$ is called invariant if a quasi-invariant probability belongs to $C$. If $C$ is invariant, the pair $(G, C)$ is called a measure groupoid.

By Theorem 2.1, every probability measure belonging to an invariant measure class is quasi-invariant. In particular, there is always a symmetric quasi-invariant probability.

Given a quasi-invariant probability measure, the quasi-invariance condition can be strengthened slightly.

LEMMA 2.4. Let $(G, C)$ be a measure groupoid, $\lambda \in C$ a probability with $r$-decomposition $\lambda=\int \lambda^{u} d \tilde{\lambda}(u)$. There is a $\tilde{\mu}$-conull Borel set $U_{0} \subset U_{G}$ such that

1. $\lambda^{u}(G)=1$ if $u \in U_{0}$.

2. $\lambda^{u}\left(G-G \mid U_{0}\right)=0$ if $u \in U_{0}$.

3. $u \in U_{0} \Rightarrow \lambda^{u}\left(r^{-1}(\{u\})\right)=1$.

4. If $x \in G \mid U_{0}$, then $x \cdot \lambda^{d(x)} \sim \lambda^{r(x)}$.

If $U \subset U_{G}$ is a conull Borel set, $(G \mid U, C)$ is called an inessential reduction (i.r.) of $(G, C)$. The term inessential contraction (i.c.) has been used in the literature. An i.r. of a measure groupoid is itself a measure groupoid.

If $\lambda \in C$ is symmetric, then $r_{*} \lambda=d_{*} \lambda$. We can also define a quasiinvariance for right translation using the $d$-decomposition $\lambda=\int \lambda_{u} d \tilde{\lambda}(u)$. However, for a.a. $u \lambda_{u}=\left(\lambda^{u}\right)^{-1}$; this formula may be used to define $\lambda_{u}$ for all $u$. From this it follows that $C$ is left invariant iff it is right invariant. Note that 
$\lambda^{(2)}=\int \lambda_{u} \times \lambda^{u} d \tilde{\lambda}(u)$ defines a measure on $G^{(2)} \cdot\left[\lambda^{(2)}\right]$ depends only on $C$ and is denoted $C^{(2)}$.

EXAMPLE 2.5. Let $g$ act on $S$ as in Example 1.2. Suppose that $g$ is locally compact second countable, that $S$ is an analytic space, and that $(s, x) \mapsto s x$ : $S \times \mathfrak{g} \rightarrow S$ is Borel. We say that the $\mathfrak{g}$ action is Borel and that $S$ is an analytic $g$-space. For $E \subset S$ let $E x=\{s x \in S: s \in E\}$. A measure $\mu$ on $S$ is called quasi-invariant for the $\mathrm{g}$ action if $\forall x \in \mathfrak{g}, E \in \mathfrak{B}(S), \mu(E x)=0$ iff $\mu(E)=0 . \mu$ is invariant if $\mu(E x)=\mu(E) \forall x \in g, E \in \mathscr{B}(S)$. If $\mu$ is quasiinvariant, $h$ a left Haar measure on $\mathrm{g}$, then $(S \times \mathrm{g},[\mu \times h])$ is a measure groupoid. $[\mu \times h]$ is symmetric because $\left[h^{-1}\right]=[h]$ and $\mu$ is quasi-invariant. The invariance of $[\mu \times h]$ follows from the invariance of $h$ under translation. One says the action of $g$ on $(S, \mu)$ is ergodic if the only Borel sets $E \subset S$ satisfying $E x=E \forall x \in g$ are either null or conull. A concept apparently weaker, that the only Borel sets $E \subset S$ such that $1_{E x}=1_{E}$ a.e. for $h$-a.a. $x \in \mathfrak{g}$ are either null or conull, is equivalent to ergodicity.

Ergodicity of $g$-actions motivates the following.

Definition 2.6. A measure groupoid $(G,[\lambda])$ is called ergodic if the only Borel functions $\phi: U_{G} \rightarrow \mathbf{R}$ satisfying $\int|\phi \circ r-\phi \circ d| d \lambda=0$ are such that $\phi=$ constant $\tilde{\lambda}$-a.e.

$(G,[\lambda])$ is ergodic iff $\forall E \in \mathscr{B}\left(U_{G}\right), \int\left|1_{E} \circ r-1_{E} \circ d\right| d \lambda=0 \Rightarrow \tilde{\lambda}(E)=0$ or $\tilde{\lambda}\left(U_{G}-E\right)=0 .(S \times \mathfrak{g},[\mu \times h])$ of Example 2.5 is ergodic iff the $g$ action on $(S, \mu)$ is ergodic.

If $E \in \mathscr{B}\left(U_{G}\right)$, the saturation $[E]=d\left(r^{-1}(E)\right)=r\left(d^{-1}(E)\right)$ is analytic, hence measurable with respect to any measure on $U_{G} .(G,[\lambda])$ is called essentially transitive if there is a $\tilde{\lambda}$-conull equivalence class $[u] \in U_{G}$. An essentially transitive measure groupoid is ergodic and has an i.r. such that any two units are equivalent.

EXAMPLE 2.7. As in Example 1.8, let $\varepsilon$ be an equivalence relation on a set $S$. Assume that $S$ is an analytic space and that $\mathcal{E} \subset S \times S$ is a Borel set. Suppose given probability measures $\mu$ and $\alpha_{s}, s \in S$, on $S$ satisfying

1. $\alpha_{s}([s])=1$ for $\mu$-a.a. $s \in S$, where $[s]=\{t:(s, t) \in \mathcal{E}\}$.

2. $E \in \mathscr{B}(S) \Rightarrow s \mapsto \alpha_{s}(E)$ is Borel.

3. $\exists \mu$-conull $S_{0} \in \mathscr{B}(S)$ such that $(s, t) \in \mathcal{E} \cap\left(S_{0} \times S_{0}\right) \Rightarrow \alpha_{s} \sim \alpha_{t}$.

4. $f \geqslant 0$ Borel on $\mathcal{E}$ and $\int f(s, t) d \alpha_{s}(t) d \mu(s)=0 \Rightarrow \int f(t, s) d \alpha_{s}(t) d \mu(s)=$ 0 . Then we say that $\left(S, \mathcal{E}, \mu,\left\{\alpha_{s}\right\}\right)$ defines a measured equivalence relation. If, in addition,

5. $E \in \mathscr{B}(S)$ and $\int\left|1_{E}(s)-1_{E}(t)\right| d \alpha_{s}(t) d \mu(s)=0 \Rightarrow \mu(E)=0$, then $\left(S, \mathcal{E}, \mu,\left\{\alpha_{s}\right\}\right)$ is an ergodic equivalence relation. Defining $\lambda(E)=$ $\int 1_{E}(s, t) d \alpha_{s}(t) d \mu(s)$ for $E \in \mathscr{B}(\mathscr{E}),(\mathcal{E},[\lambda])$ is a principal measure groupoid and is ergodic iff the equivalence relation is ergodic. Conversely, if $(G,[\lambda])$ is a principal groupoid, the measures $\lambda^{u}$ in the $r$-decomposition of the probability $\lambda$ are supported on $\{u\} \times[u]$, so they are of the form $\delta_{u} \times \alpha_{u}$. Then 
$\left(U_{G}, G, \tilde{\lambda},\left\{\alpha_{u}\right\}\right)$ is a measured equivalence relation.

Example 2.8. Let $(G, C)$ be a measure groupoid. Then if $\lambda \in C$ is a probability, $\left((r, d)(G),\left[(r, d)_{*} \lambda\right]\right)$ is a principal groupoid. $\left[(r, d)_{*} \lambda\right]$ does not depend upon the choice of the probability $\lambda .\left((r, d)(G),\left[(r, d)_{*} \lambda\right]\right)$ is ergodic iff $(G, C)$ is. $\left((r, d)_{*} \lambda\right)^{\sim}=\tilde{\lambda}$. See [14], [17].

Definition 2.9. A Borel function $\rho$ from a measure groupoid $(G, C)$ into a Borel groupoid $H$ is a strict homomorphism if $\rho$ satisfies Definition 1.9. $\rho$ is a homomorphism if there is an i.r. $G_{0}$ of $G$ such that $\rho \mid G_{0}$ is a strict homomorphism. $\rho$ is an a.e. homomorphism if $\left\{(x, y) \in G^{(2)}:(\rho(x), \rho(y)) \in\right.$ $H^{(2)}$ and $\left.\rho(x y)=\rho(x) \rho(y)\right\}$ is $C^{(2)}$-conull.

As Ramsay has observed [14, p. 58], the following is an immediate consequence of the proofs of Theorem 5.1 and Lemma 5.2 of [13].

THEOREM 2.10. Let $(G, C)$ be a measure groupoid and $H$ an analytic Borel groupoid. Let $\rho: G \rightarrow H$ be an a.e. homomorphism. There is an i.r. $G_{0}$ of $G$ and $a$ Borel function $\rho_{0}: G \rightarrow H$ such that $\rho_{0}=\rho$ a.e. and $\rho_{0} \mid G_{0}$ is a strict homomorphism. Furthermore, if $F \subset G$ is conull and $\left\{x y \in G:(x, y) \in G^{(2)}\right.$ $\cap(F \times F)\} \subset F, F$ contains an i.r.

3. Existence of Haar measure. Let $(G, C)$ be a measure groupoid, $\lambda \in C$ a symmetric probability with $r$-decomposition $\lambda=\int \lambda^{u} d \tilde{\lambda}(u)$. Let $U_{0} \subset U_{G}$ be as in Lemma 2.4 and let $G_{0}=G \mid U_{0}$. The quasi-invariance of $\lambda$ means that

$$
\left(f \mapsto \int f(y) d \lambda^{r(x)}(y)\right) \sim\left(f \mapsto \int f(x y) d \lambda^{d(x)}(y)\right) ;
$$

that is, these integrals have the same null functions. Referring to Example 2.5, we see that, at least for certain groupoids, the quasi-invariance can be replaced by invariance if we are willing to replace $\lambda$ by an equivalent measure not necessarily finite or symmetric. Specifically, on $S \times g$ the measure $\mu \times h$ has $r$-decomposition $\mu \times h=\int\left(\delta_{s} \times h\right) d \mu(s)$. The measures $\delta_{s} \times h$ satisfy

$$
\begin{aligned}
& \int f(t, y) d\left(\delta_{s} \times h\right)(t, y)=\int f(s, y) d h(y)=\int f(s, x y) d h(y) \\
& =\int f((s, x)(s x, y)) d h(y)=\int f((s, x)(t, y)) d\left(\delta_{s x} \times h\right)(t, y) .
\end{aligned}
$$

If $\nu \sim \lambda$ has $r$-decomposition $\nu=\int \nu^{u} d \tilde{\lambda}(u)$ with respect to $\tilde{\lambda}$ on $G_{0}$, the generalization (3.1) to the abstract groupoid is

$$
\int f(y) d \nu^{r(y)}(y)=\int f(x y) d \nu^{d(x)}(y) .
$$

In terms of the measured equivalence relation $\left(S, \mathcal{E}, \mu,\left\{\alpha_{s}\right\}\right)$, an invariant measure $\nu$ has been found if we can find $\sigma$-finite measures $\beta_{s} \sim \alpha_{s}$ such that $s \mapsto \delta_{s} \times \beta_{s}(E)$ is Borel and $s \sim t \Rightarrow \beta_{s}=\beta_{t}$. The main theorem of this 
section asserts that every measure groupoid has a measure $\nu$ satisfying (3.2) for $x$ in an i.r.

Recall the groupoid structure on $G^{(2)}$ in Example 1.3.

LEMMA 3.3. If $G$ is an analytic (standard) Borel groupoid, $G^{(2)} \subset G \times G$ is an analytic (standard) Borel groupoid.

Proof. $G \times G$ is analytic (standard) if $G$ is. By definition of Borel groupoid, $G^{(2)} \subset G \times G$ is Borel, hence also analytic (standard). $G^{(2)(2)}=$ $\left\{((x, y),(z, w)) \in G^{(2)} \times G^{(2)}: x y=z\right\}$ is the set where two Borel functions to a countably separated space agree. Thus $G^{(2)(2)}$ is Borel.

$(x, y) \mapsto(x, y)^{-1}=\left(x y, y^{-1}\right)$ is Borel on $G^{(2)}$ because $(x, y) \mapsto(x y)$ and $(x, y) \mapsto y^{-1}$ are Borel from $G^{(2)}$ to $G . G^{(2)(2)} \subset\left\{(x, y, z, w) \in G^{4}: d(y)=\right.$ $r(w)\}$, on which $(x, y, z, w) \mapsto(x, y w)$ is well defined and Borel. Thus multiplication in $G^{(2)}$ is Borel.

Proposition 3.4. If $(G, C)$ is an analytic groupoid with invariant measure class, so is $\left(G^{(2)}, C^{(2)}\right)$.

Proof. Choose $\lambda=\int \lambda^{u} d \tilde{\lambda}(u) \in C$, a symmetric probability. Let $\lambda_{u}=$ $\left(\lambda^{u}\right)^{-1}$. Suppose $f$ is a nonnegative Borel function such that $\int f(x y$, $\left.y^{-1}\right) d \lambda^{(2)}(x, y)=0$. Then

$$
\iint f\left(x y, y^{-1}\right) d \lambda_{r(y)}(x) d \lambda(y)=0
$$

so $\int f\left(x y, y^{-1}\right) d \lambda_{r(y)}(x)=0$ for $\lambda$-a.a. $y$. Since $[\lambda]$ is invariant, $g \mapsto$ $\int g(x y) d \lambda_{r(y)}(x)$ and $g \mapsto \int g(x) d \lambda_{d(y)}(x)$ are equivalent for a.a $y$. Hence $\int f\left(x, y^{-1}\right) d \lambda_{d(y)}(x)=0$ for a.a. $y$, so

$$
\iint f\left(x, y^{-1}\right) d \lambda_{d(y)}(x) d \lambda(y)=0 .
$$

Because $\lambda$ is symmetric, $\iint f(x, y) d \lambda_{r(y)}(x) d \lambda(y)=0$. Thus

$$
0=\iiint f(x, y) d \lambda_{u}(x) d \lambda^{u}(y) d \lambda(u)=\int f(x, y) d \lambda^{(2)}(x, y) .
$$

Conversely, if $\int f(x, y) d \lambda^{(2)}(x, y)=0, \int f\left(x y, y^{-1}\right) d \lambda^{(2)}(x, y)=0$, so $\left[\lambda^{(2)}\right]$ is symmetric. Via the maps $x \mapsto(x, d(x))$ and $(x, u) \mapsto x$ we may identify $G$ with $U_{G^{(2)}}=(G \times U) \cap G^{(2)}$. Then since

$$
\begin{aligned}
\int f\left(r^{(2)}(x, y)\right) d \lambda^{(2)}(x, y) & =\int f(x, d(x)) d \lambda_{u}(x) d \lambda^{u}(y) d \tilde{\lambda}(u) \\
& =\int f(x, d(x)) d \lambda_{u}(x) d \tilde{\lambda}(u) \\
& =\int f(x, d(x)) d \lambda(x),
\end{aligned}
$$


$r_{*}^{(2)}\left(\lambda^{(2)}\right)=\lambda$. The $r^{(2)}$-decomposition, $\lambda^{(2)}=\int \lambda^{(2)(x, d(x))} d \lambda(x)$, of $\lambda^{(2)}$ is thus $\lambda^{(2)}=\int \delta_{x} \times \lambda^{d(x)} d \lambda(x)$. That is, $\lambda^{(2)(x, d(x))}=\delta_{x} \times \lambda^{d(x)}$.

Let $U_{0} \subset U_{G}$ be a conull Borel set such that

$$
x \in G \mid U_{0} \Rightarrow g \mapsto \int g(y) d \lambda^{r(x)}(y) \sim g \mapsto \int g(x y) d \lambda^{d(x)}(y) .
$$

Let $(x, y) \in G^{(2)}\left|\left(G \mid U_{0}\right) . d^{(2)}(x, y) \in G\right| U_{0}$ and $r^{(2)}(x, y) \in G \mid U_{0}$, so $x y \in$ $G \mid U_{0}$ and $x \in G \mid U_{0}$. Hence $y \in G \mid U_{0}$.

$$
\begin{gathered}
\int f((x, y)(z, w)) d \lambda^{(2) d^{(2)}(x, y)}(z, w) \\
=\int f((x, y)(z, w)) d \lambda^{(2)(x y, d(y))}(z, w) \\
=\int f((x, y)(z, w)) d \delta_{x y} \times \lambda^{d(y)}(z, w) \\
=\int f(x, y w) d \lambda^{d(y)}(w) . \\
\int f(z, w) d \lambda^{(2) r^{(2)}(x, y)}(z, w)=\int f(z, w) d \lambda^{(2)(x, d(x))}(z, w) \\
=\int f(z, w) d \delta_{x} \times \lambda^{d(x)}(z, w) \\
=\int f(x, w) d \lambda^{d(x)}(w) \\
=\int f(x, w) d \lambda^{r(y)}(w) .
\end{gathered}
$$

Since $y \in G \mid U_{0}$, the integrals

$$
f \mapsto \int f(x, y w) d \lambda^{d(y)}(w) \text { and } f \mapsto \int f(x, w) d \lambda^{r(y)}(w)
$$

are equivalent. Thus $\left[\lambda^{(2)}\right]$ is invariant.

REMARK 3.5. $\left(G^{(2)}, C^{(2)}\right)$ is ergodic iff there is a single unit $u_{0} \in U_{G}$ such that $U_{G}-\left\{u_{0}\right\}$ is null. Indeed, if $\phi$ is any Borel function on $U_{G}$, then $g(x, d(x))=\phi \circ r(x)$ satisfies $g \circ r^{(2)}=g \circ d^{(2)}$. Conversely, these are the

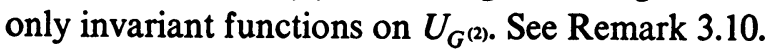

Let $\tau(x, y)=(x, y)^{-1}=\left(x y, y^{-1}\right)$. Since $\left[\lambda^{(2)}\right]$ is symmetric, $\tau_{*} \lambda^{(2)} \sim \lambda^{(2)}$, so there is a Borel function $\rho$ such that $0<\rho<\infty$ and

$$
\int f(x, y) \rho(x, y) d \lambda^{(2)}(x, y)=\int f\left(x y, y^{-1}\right) d \lambda^{(2)}(x, y) .
$$

LEMMA 3.6. $\rho$ is an a.e. homomorphism of $G^{(2)}$ into $\mathbf{R}_{+}^{*}$, the group of positive real numbers under multiplication; and there is a conull Borel set $G_{0} \subset G$ such that $x \in G_{0}$ and $E \in \mathscr{B}(G)$ imply 


$$
\int 1_{E}(x y) d \lambda^{d(x)}(y)=\int 1_{E}(y) \rho\left(y^{-1}, x\right) d \lambda^{r(x)}(y) .
$$

Proof. Let $E, F \in \Re(G)$.

$$
\begin{aligned}
\int 1_{E}(y) & 1_{F}\left(x^{-1}\right) \rho\left(y, x^{-1}\right) d \lambda_{d(x)}(y) d \lambda(x) \\
& =\int 1_{E \times F}(y, x) \rho(y, x) d \lambda^{(2)}(y, x) \\
& =\int 1_{E \times F}\left(y x, x^{-1}\right) d \lambda_{u}(y) d \lambda^{u}(x) d \tilde{\lambda}(u) \\
& =\int 1_{E}(y x) 1_{F}\left(x^{-1}\right) d \lambda_{r(x)}(y) d \lambda(x) .
\end{aligned}
$$

Therefore for $\lambda$-a.a. $x$,

$$
\begin{aligned}
\int 1_{E}(y x) d \lambda_{r(x)}(y) & =\int 1_{E}(y) \rho\left(y, x^{-1}\right) d \lambda_{d(x)}(y), \\
\int 1_{E^{-1}}\left(x^{-1} y\right) d \lambda^{r(x)}(y) & =\int 1_{E^{-1}}(y) \rho\left(y^{-1}, x^{-1}\right) d \lambda^{d(x)}(y), \\
\int 1_{E^{-1}}(x y) d \lambda^{d(x)}(y) & =\int 1_{E^{-1}}(y) \rho\left(y^{-1}, x\right) d \lambda^{r(x)}(y) .
\end{aligned}
$$

Let $Q$ be a countable algebra generating $\mathscr{B}(G)$. There is a conull Borel set $G_{0} \subset G$ such that $x \in G_{0}$ and $E \in \mathbb{Q}$ imply

$$
\int 1_{E}(x y) d \lambda^{d(x)}(y)=\int 1_{E}(y) \rho\left(y^{-1}, x\right) d \lambda^{r(x)}(y) .
$$

By Caratheodory extension, the equation holds for $x \in G_{0}$ and $E \in \mathscr{B}(G)$. Suppose now that $x, y$ and $x y$ belong to $G_{0}$. Then

$$
\begin{aligned}
\int f(z) \rho\left(z^{-1}, x y\right) d \lambda^{r(x y)}(z) & =\int f(x y z) d \lambda^{d(x y)}(z) \\
& =\int f(x z) \rho\left(z^{-1}, y\right) d \lambda^{d(x)}(z) \\
& =\int f(z) \rho\left(z^{-1}, x\right) \rho\left(z^{-1} x, y\right) d \lambda^{r(x y)}(z)
\end{aligned}
$$

since $\rho\left(z^{-1}, y\right)=\rho\left((x z)^{-1} x, y\right)$, so $z \mapsto\left|\rho\left(z^{-1}, x y\right)-\rho\left(z^{-1}, x\right) \rho\left(z^{-1} x, y\right)\right|$ is $\lambda^{r(x)}$-null.

$$
\begin{aligned}
\int 1_{G-G_{0}}(x y) d \lambda^{(2)}(x, y) & =\int 1_{G-G_{0}} \circ r^{(2)} \circ \tau(x, y) d \lambda^{(2)}(x, y) \\
& =\int 1_{G-G_{0}}(x) \rho(x, y) d \lambda^{(2)}(x, y) \\
& =\int 1_{G-G_{0}}(x) \rho(x, y) d \lambda^{d(x)}(y) d \lambda(x) \\
& =0 \text { since } G_{0} \text { is } \lambda \text {-conull. }
\end{aligned}
$$


Hence $(x, y) \mapsto 1_{G_{0}}(x y)$ is 1 a.e., so $(x, y) \mapsto 1_{G_{0}}(x y) 1_{G_{0}}(x) 1_{G_{0}}(y)$ is 1 a.e. That is, for $\lambda^{(2)}$-a.a. $(x, y), x, y$, and $x y$ belong to $G_{0}$. Thus, for $\lambda^{(2)}$-a.a. $(x, y)$, $z \mapsto\left|\rho\left(z^{-1}, x y\right)-\rho\left(z^{-1}, x\right) \rho\left(z^{-1} x, y\right)\right|$ is $\lambda^{r(x)}$-null. The measure $\lambda^{(2)(2)}$ on $G^{(2)(2)}$ is

$$
\int\left(\tau_{*} \lambda^{(2)(x, d(x))} \times \lambda^{(2)(x, d(x))}\right) d \lambda(x)
$$

so

$$
\begin{aligned}
\int F((s, t), & (v, w)) d \lambda^{(2)(2)}((s, t),(v, w)) \\
& =\iiint F\left(\left(x t, t^{-1}\right),(x, w)\right) d \lambda^{d(x)}(t) d \lambda^{d(x)}(w) d \lambda(x) .
\end{aligned}
$$

To show that $\rho$ is a $\lambda^{(2)(2)}$-a.e. homomorphism we must show

$$
0=\int|\rho(s, t) \rho(v, w)-\rho((s, t)(v, w))| d \lambda^{(2)(2)}((s, t),(v, w)),
$$

so we must show that

$$
c=\iiint\left|\rho\left(x t, t^{-1}\right) \rho(x, w)-\rho\left(x t, t^{-1} w\right)\right| d \lambda^{d(x)}(t) d \lambda^{d(x)}(w) d \lambda(x)=0 .
$$

But

$$
\begin{gathered}
c=\iiint \mid \rho\left(\left(t^{-1} x\right)^{-1}, t^{-1}\right) \rho\left(x^{-1}, w\right) \\
-\rho\left(\left(t^{-1} x\right)^{-1}, t^{-1} w\right) \mid d \lambda^{r(t)}(w) d \lambda^{r(t)}(x) d \lambda(t) \\
=\iiint\left|\rho\left(x^{-1}, t^{-1}\right) \rho\left(x^{-1} t^{-1}, w\right)-\rho\left(x^{-1}, t^{-1} w\right)\right| \\
\cdot \rho\left(x^{-1}, t^{-1}\right) d \lambda^{r(t)}(w) d \lambda^{d(t)}(x) d \lambda(t) \\
=\iiint\left|\rho\left(x^{-1}, t\right) \rho\left(x^{-1} t, w\right)-\rho\left(x^{-1}, t w\right)\right| \\
\cdot \cdot \rho\left(x^{-1}, t\right) d \lambda^{r(t)}(x) d \lambda^{d(t)}(w) d \lambda(t) \\
=\int\left(\int\left|\rho\left(x^{-1}, t w\right)-\rho\left(x^{-1}, t\right) \rho\left(x^{-1} t, w\right)\right|\right. \\
\left.\quad \cdot \rho\left(x^{-1}, t\right) d \lambda^{r(t)}(x)\right) d \lambda^{(2)}(t, w) \\
=0 .
\end{gathered}
$$

Therefore, $\rho$ is a $\lambda^{(2)(2)}$-a.e. homomorphism.

By Theorem 2.10, there is a conull Borel set $G_{1} \subset G$ such that $\rho$ agrees a.e. on $G^{(2)} \mid G_{1}$ with a strict homomorphism of $G^{(2)} \mid G_{1}$ into $\mathbf{R}_{+}^{*}$, and this homomorphism is Borel. By modifying $\rho$ on a null Borel set, we may assume that $\rho$ itself is a strict homomorphism on $G^{(2)} \mid G_{1}$. Reapplying the preceding 
lemma, using this new $\rho$, there is a conull Borel $G_{2}$ such that $x \in G_{2}$ and $f \geqslant 0$ Borel on $G$ imply

$$
\int f(x y) d \lambda^{d(x)}(y)=\int f(y) \rho\left(y^{-1}, x\right) d \lambda^{r(x)}(y) .
$$

The fact that $\rho \mid\left(G^{(2)} \mid G_{1}\right)$ is a strict homomorphism means that $x, x y$, and $x y z \in G_{1} \Rightarrow \rho(x, y) \rho(x y, z)=\rho(x, y z)$.

We are prepared now to prove the existence of an invariant or "Haar" measure in the invariant measure class $C$ of an analytic groupoid $G$.

THEOREM 3.7. Let $(G, C)$ be a measure groupoid, $\sigma$ any $C^{(2)(2)}$-a.e. homomorphism of $G^{(2)}$ into an analytic group $A$. There is a Borel function $p$ : $G \rightarrow A$ such that for $C^{(2)}-a . a .(x, y), \sigma(x, y)=p(x) p(x y)^{-1}$. Consequently, if $\lambda \in C$ is a symmetric probability with $r$-decomposition $\lambda=\int \lambda^{u} d \tilde{\lambda}(u)$, there is a conull Borel set $U_{0} \subset U_{G}$ and a positive Borel function $P$ on $G$ such that for every $u \in U_{0}, \lambda^{u}(G)=\lambda^{u}\left(G \mid U_{0}\right)=1$, and for every $x \in G \mid U_{0}$ and every Borel function $f \geqslant 0$ on $G$,

$$
\int f(x y) P(y) d \lambda^{d(x)}(y)=\int f(y) P(y) d \lambda^{r(x)}(y) .
$$

Proof. The first assertion says that the first cohomology of $G^{(2)}$ is trivial, even for a.e. cocycles. Applying Theorem 2.10, we may assume that there is a $C$-null Borel set $G_{0} \subset G$ such that $\sigma$ is strict on $G^{(2)} \mid G_{0}$. Let $U_{1}=\left\{u \in U_{G}\right.$ : $\left.\lambda^{u}\left(G_{0}\right)=1\right\}$. By the von Neumann Selection Lemma [1, p. 12], we find a conull Borel set $U_{2} \subset U_{1}$ and Borel function $\theta: U_{2} \rightarrow G_{0}$ such that $r \circ \theta=$ identity. Let $\phi=\theta \circ r$ on $G-G_{0}$ and $\phi=$ identity on $G_{0}$. Define $\sigma^{\prime}(x, y)=$ $\sigma\left(\phi(x), \phi(x)^{-1} \phi(x y)\right)$ for $(x, y) \in G^{(2)} \mid\left(G \mid U_{2}\right)$. Since

$$
d\left(\phi(x)^{-1}\right)=r(\phi(x))=r(x)=r(\phi(x y)),
$$

$\sigma^{\prime}$ is well defined. If $(x, y) \in G^{(2)} \mid\left(\left(G \mid U_{2}\right) \cap G_{0}\right)$, then $x \in G_{0}$ and $x y \in G_{0}$, so $\sigma^{\prime}(x, y)=\sigma(x, y)$. Thus $\sigma=\sigma^{\prime} C^{(2)}$-a.e. If $(x, y) \in G^{(2)} \mid\left(G \mid U_{2}\right)$ and $(x y, z)$ $\in G^{(2)} \mid\left(G \mid U_{2}\right)$, then

$$
\begin{aligned}
\sigma^{\prime}(x, y) \sigma^{\prime}(x y, z) & =\sigma\left(\phi(x), \phi(x)^{-1} \phi(x y)\right) \sigma\left(\phi(x y), \phi(x y)^{-1} \phi(x y z)\right) \\
& =\sigma\left(\phi(x), \phi(x)^{-1} \phi(x y z)\right)=\sigma^{\prime}(x, y z)
\end{aligned}
$$

because $\phi(x), \phi(x y)$, and $\phi(x y z)$ belong to $G_{0}$. Thus $\sigma^{\prime}$ is a strict homomorphism on $G^{(2)} \mid\left(G \mid U_{2}\right)$. If $x \in G \mid U_{2}$ and $y \in G \mid U_{2}$, and $d(x)=$ $r(y)$, then $x, x y$, and $x y\left(y^{-1} x^{-1}\right)$ belong to $G \mid U_{2}$; and from $\sigma^{\prime}(x, y) \sigma^{\prime}(x y$, $\left.y^{-1} x^{-1}\right)=\sigma^{\prime}\left(x, y\left(y^{-1} x^{-1}\right)\right)$ we obtain

$$
\sigma^{\prime}(x, y)=\sigma^{\prime}\left(x, x^{-1}\right) \sigma^{\prime}\left(x y, y^{-1} x^{-1}\right)^{-1} .
$$

Let $p(x)=\sigma^{\prime}\left(x, x^{-1}\right)$.

For $\sigma=\rho$, we find $\rho(x, y)=p(x) / p(x y) \lambda^{(2)}$-a.e. Applying Lemma 3.6 to 
$(x, y) \mapsto p(x) / p(x y)$, we obtain a conull Borel $G_{0} \subset G$ such that $x \in G_{0}$ implies

$$
\begin{aligned}
\int f(x y) p\left(y^{-1}\right) d \lambda^{d(x)}(y) & =\int f(y) p\left(y^{-1} x\right) p\left(y^{-1}\right) p\left(y^{-1} x\right)^{-1} d \lambda^{r(x)}(y) \\
& =\int f(y) p\left(y^{-1}\right) d \lambda^{r(x)}(y)
\end{aligned}
$$

for $f \geq 0$ Borel. Let $P(y)=p\left(y^{-1}\right)$.

$$
F=\left\{x \in G: \int f(x y) P(y) d \lambda^{d(x)}(y)=\int f(y) P(y) d \lambda^{r(x)}(y)\right\}
$$

is conull and multiplicative. By Theorem 2.10, $F$ contains an i.r. Application of Lemma 2.4 completes the proof.

THEOREM 3.8. The modular function $\Delta=\left(y \mapsto P(y) / P\left(y^{-1}\right)\right)$ is a $\lambda^{(2)}$-a.e. homomorphism.

Proof. We may assume that $G$ has been replaced by $G \mid U_{0}$ of the preceding theorem, since

$$
\lambda^{(2)}\left(\left(G\left|U_{0} \times G\right| U_{0}\right) \cap G^{(2)}\right)=1 .
$$

Let $E$ and $F$ be Borel subsets of $G$ and define $f=1_{E} / P, g=1_{F} / P$, $f^{*}=\left(y \mapsto f\left(y^{-1}\right) \Delta\left(y^{-1}\right)\right)$, and $g^{*}=\left(y \mapsto g\left(y^{-1}\right) \Delta\left(y^{-1}\right)\right)$. Note that $f^{*}(y)$ $=1_{E^{-1}}(y) / P(y)$ and $g^{*}(y)=1_{F^{-1}}(y) / P(y)$. Define a bounded operator $T_{f}$ (and similarly $T_{g}, T_{f^{*}}, T_{g^{*}}$ ) by

$$
\begin{aligned}
\left(T_{f} \phi, \psi\right) & =\int f(y) \phi(d(y)) \overline{\psi(r(y))} P(y) d \lambda(y) \\
& =\int_{E} \phi(d(y)) \overline{\psi(r(y))} d \lambda(y) \\
& =\int\left(\int f(y) \phi(d(y)) P(y) d \lambda^{u}(y)\right) \overline{\psi(u)} d \tilde{\lambda}(y)
\end{aligned}
$$

for $\phi, \psi \in L^{2}\left(U_{G}, \tilde{\lambda}\right)$.

$$
\begin{aligned}
\left|\left(T_{f} \phi, \psi\right)\right| & \leqslant \int|\phi(d(y))||\psi(r(y))| d \lambda(y) \\
& \leqslant \int|\phi(d(y))|^{2} d \lambda(y)^{1 / 2} \int|\psi(r(y))|^{2} d \lambda(y)^{1 / 2} \\
& =\int|\phi(u)|^{2} d \tilde{\lambda}(u)^{1 / 2} \int|\psi(u)|^{2} d \tilde{\lambda}(u)^{1 / 2} \\
& =\|\phi\|_{2}\|\psi\|_{2} .
\end{aligned}
$$

Since 


$$
\begin{aligned}
& \int\left(\int f(y) \phi(d(y)) P(y) d \lambda^{u}(y)\right) \overline{\psi(u)} d \tilde{\lambda}(u) \\
& \quad=\left(T_{f} \phi, \psi\right)=\int T_{f} \phi(u) \overline{\psi(u)} d \tilde{\lambda}(u) \text { for all } \psi \in L^{2}\left(U_{G}, \tilde{\lambda}\right),
\end{aligned}
$$

we conclude that

$$
T_{f} \phi=\left(u \mapsto \int f(y) \phi(d(y)) P(y) d \lambda^{u}(y)\right) \tilde{\lambda} \text {-a.e. }
$$

The same is true of $g, f^{*}$, and $g^{*}$.

$$
\begin{aligned}
\int f^{*}(y) \phi( & (y(y)) \overline{\psi(r(y))} P(y) d \lambda(y) \\
& =\int f\left(y^{-1}\right) \phi(d(y)) \overline{\psi(r(y))} P\left(y^{-1}\right) d \lambda(y) \\
& =\int \overline{f(y) \psi(d(y))} \phi(r(y)) P(y) d \lambda(y) \\
& =\overline{\left(T_{f} \psi, \phi\right)}=\left(\phi, T_{f} \psi\right)=\left(T_{f}^{*} \phi, \psi\right) .
\end{aligned}
$$

Hence $T_{f^{*}}=T_{f}^{*}$ and also $T_{g^{*}}=T_{g}^{*}$.

$$
\begin{aligned}
\left(T_{f} T_{g} 1,1\right) & =\int f(x)\left(\int g(y) P(y) d \lambda^{d(x)}(y)\right) P(x) d \lambda(x) \\
& =\iint f(x)\left(\int g\left(x^{-1} y\right) P(y) d \lambda^{r(x)}(y)\right) P(x) d \lambda^{u}(x) d \tilde{\lambda}(u) \\
& =\iint f(x)\left(\int g\left(x^{-1} y\right) P(y) d \lambda^{u}(y)\right) P(x) d \lambda^{u}(x) d \tilde{\lambda}(u) \\
& =\iiint f(x) g\left(x^{-1} y\right) P(x) P(y) d \lambda^{r(y)}(x) d \lambda^{u}(y) d \tilde{\lambda}(u) \\
& =\iiint f(y x) g\left(x^{-1}\right) P(x) P(y) d \lambda^{d(y)}(x) d \lambda^{u}(y) d \tilde{\lambda}(u) \\
& =\iint f(x y) g\left(y^{-1}\right) P(y) d \lambda^{d(x)}(y) P(x) d \lambda(x) .
\end{aligned}
$$

Applying this calculation to $T_{g^{*}} T_{f^{*}}$, we have

$$
\begin{aligned}
\iint g^{*}(x y) & f^{*}\left(y^{-1}\right) P(y) d \lambda^{d(x)}(y) P(x) d \lambda(x) \\
= & \left(T_{g^{*}} T_{f^{*}} 1,1\right)=\left(1, T_{f} T_{g} 1\right)=\overline{\left(T_{f} T_{g} 1,1\right)} \\
= & \iint f(x y) g\left(y^{-1}\right) P(y) d \lambda^{d(x)}(y) P(x) d \lambda(x) \\
= & \left.\quad \text { (using } x \mapsto x^{-1}\right) \\
= & \iint f\left(x^{-1} y\right) g\left(y^{-1}\right) P(y) d \lambda^{r(x)}(y) \Delta(x)^{-1} P(x) d \lambda(x)
\end{aligned}
$$


Since

$$
\begin{aligned}
& \iint g^{*}(x y) f^{*}\left(y^{-1}\right) P(y) d \lambda^{d(x)}(y) P(x) d \lambda(x) \\
& \quad=\iint f(y) g\left(y^{-1} x^{-1}\right) \Delta(y) \Delta\left(y^{-1} x^{-1}\right) P(y) d \lambda^{d(x)}(y) P(x) d \lambda(x),
\end{aligned}
$$

we have

$$
\begin{aligned}
& \iint f(y) g\left(y^{-1} x^{-1}\right) P(y) P(x) \\
& \cdot\left(\Delta(y) \Delta\left(y^{-1} x^{-1}\right)-\Delta(x)^{-1}\right) d \lambda^{d(x)}(y) d \lambda(x)=0
\end{aligned}
$$

and the integrand is absolutely integrable. Since $\Delta(x)^{-1}=\Delta\left(x^{-1}\right)$,

$$
\begin{aligned}
& 0=\iint f(y) g\left(y^{-1} x^{-1}\right) P(x) P(y) \\
& \cdot\left(\Delta(y) \Delta(x y)^{-1}-\Delta(x)^{-1}\right) d \lambda^{d(x)}(y) d \lambda(x) \\
& =\iiint f\left(x^{-1} y\right) g\left(y^{-1}\right) P(x) P(y) \\
& \cdot\left(\Delta\left(x^{-1} y\right) \Delta(y)^{-1}-\Delta(x)^{-1}\right) d \lambda^{r(x)}(y) d \lambda^{u}(x) d \tilde{\lambda}(u) \\
& =\iiint f\left(\left(y^{-1} x\right)^{-1}\right) g\left(y^{-1}\right) P(x) P(y) \\
& \cdot\left(\Delta\left(y^{-1} x\right)^{-1} \Delta(y)^{-1}-\Delta(x)^{-1}\right) d \lambda^{u}(x) d \lambda^{u}(y) d \tilde{\lambda}(u) \\
& =\iiint f\left(x^{-1}\right) g\left(y^{-1}\right) P(x) P(y) \\
& \cdot\left(\Delta(x)^{-1} \Delta(y)^{-1}-\Delta(y x)^{-1}\right) d \lambda^{d(y)}(x) d \lambda_{u}(y) d \tilde{\lambda}(u) \\
& =\iiint f(x) g(y) P\left(x^{-1}\right) P\left(y^{-1}\right) \\
& \text { - }(\Delta(x) \Delta(y)-\Delta(x y)) d \lambda_{r(y)}(x) d \lambda^{u}(y) d \tilde{\lambda}(u) \\
& =\iiint 1_{E}(x) 1_{F}(y) \Delta(x)^{-1} \Delta(y)^{-1} \\
& \cdot(\Delta(x) \Delta(y)-\Delta(x y)) d \lambda_{u}(x) d \lambda^{u}(y) d \tilde{\lambda}(u) \\
& =\int 1_{E \times F}(x, y)\left(1-\frac{\Delta(x y)}{\Delta(x) \Delta(y)}\right) d \lambda^{(2)}(x, y) .
\end{aligned}
$$

The absolute integrability of the integrand implies that $(x, y) \mapsto 1-$ $\Delta(x y) / \Delta(x) \Delta(y)$ is $\lambda^{(2)}$-absolutely integrable.

By the Carathéodory Extension Theorem, $(x, y) \mapsto(1-$ $\left.\Delta(x y) \Delta(x)^{-1} \Delta(y)^{-1}\right)$ is $\lambda^{(2)}$-null. Therefore, $\Delta(x y)=\Delta(x) \Delta(y)$ for $\lambda^{(2)}$-a.a. $(x, y) \in G^{(2)}$. 
THEOREM 3.9. Let $(G, C)$ be an analytic groupoid with invariant measure class and $\lambda \in C$ a symmetric probability. There is a conull Borel set $U_{0} \subset U_{G}$ and $a$ Borel function $P: G \mid U_{0} \rightarrow \mathbf{R}_{+}-\{0\}$ such that:

1. $\lambda$ has an $r$-decomposition $\lambda=\int \lambda^{u} d \tilde{\lambda}(u)$ on $G_{0}=G \mid U_{0}$ such that $\lambda^{u}\left(G_{0}\right)$ $=1$ for all $u \in U_{0}$.

2. For all Borel $f: G_{0} \rightarrow \mathbf{R}_{+}$and all $x \in G_{0}$,

$$
\int f(x y) P(y) d \lambda^{d(x)}(y)=\int f(y) P(y) d \lambda^{r(x)}(y) .
$$

3. $y \mapsto P(y) / P\left(y^{-1}\right)$ is a strict homomorphism of $G_{0}$ into $\mathbf{R}_{+}^{*}$. Furthermore, if $P^{\prime}$ and $U_{0}^{\prime}$ have these properties, there is a Borel function $\phi$ : $U_{0} \cap U_{0}^{\prime} \rightarrow \mathbf{R}_{+}-\{0\}$ such that $P^{\prime}(y)=\phi(d(y)) P(y)$ for $\lambda$-a.a.y.

Proof. By Theorem 3.7, there are $P_{1}$ and $U_{1}$ for which properties 1 and 2 hold; by Theorem 3.8, $y \rightarrow P_{1}(y) / P_{1}\left(y^{-1}\right)$ is a $\lambda^{(2)}$-a.e. homomorphism. By Theorem 2.10 there is an inessential reduction $G_{2}=G \mid U_{2}$ of $G \mid U_{1}$ on which is defined a strict Borel homomorphism $\Delta$ such that $P_{1}(y) / P_{1}\left(y^{-1}\right)=\Delta(y)$ for $\lambda$-a.a. $y$. Let $F=\left\{y \in G_{2}: P_{1}(y) \neq \Delta(y) P_{1}\left(y^{-1}\right)\right\} . F=F^{-1}$ is null and Borel. Define

$$
P(y)=\Delta(y)^{1 / 2} \text { for } y \in F,=P_{1}(y) \text { for } y \in G_{2}-F .
$$

On $F$,

$$
P(y) / P\left(y^{-1}\right)=\Delta(y)^{1 / 2} / \Delta\left(y^{-1}\right)^{1 / 2}=\Delta(y)
$$

and on $G_{2}-F, P(y) / P\left(y^{-1}\right)=\Delta(y)$ also. $P$ is Borel. Let $U_{3}=\left\{u \in U_{2}\right.$ : $\left.\int\left|P(y)-P_{1}(y)\right| d \lambda^{u}(y)=0\right\}$. Since $u \mapsto \int\left|P(y)-P_{1}(y)\right| d \lambda^{u}(y)$ is Borel, $U_{3}$ is Borel. Since $\int\left|P(y)-P_{1}(y)\right| d \lambda(y)=0, U_{3}$ is $\tilde{\lambda}$-conull. As before, by Lemma 2.4, there is a conull Borel $U_{0} \subset U_{3}$ such that $u \in U_{0} \Rightarrow \lambda^{u}\left(G \mid U_{0}\right)=$ 1.

$\int f(y) P(y) d \lambda^{u}(y)=\int f(y) P_{1}(y) d \lambda^{u}(y)$ for all $u \in U_{0}$ and $f$ Borel on $G \mid U_{0}$, so $U_{0}$ and $P$ satisfy the three properties.

If $U_{0}^{\prime}$ and $P^{\prime}$ do, too, we can find an i.r. $G \mid U_{4}$ and $r$-decomposition $\lambda=\int \lambda^{u} d \tilde{\lambda}(u)$ such that both $\left(P, U_{4}\right)$ and $\left(P^{\prime}, U_{4}\right)$ satisfy the three properties. Then

$$
\begin{aligned}
\int f(y) P^{\prime}(y) P\left(x^{-1} y\right) d \lambda^{r(x)}(y) & =\int f(x y) P^{\prime}(y) P(y) d \lambda^{d(x)}(y) \\
& =\int f(y) P^{\prime}\left(x^{-1} y\right) P(y) d \lambda^{r(x)}(y)
\end{aligned}
$$

for $x \in G \mid U_{4}, f \geqslant 0$ Borel on $G \mid U_{4} \cdot R(y)=P(y) / P^{\prime}(y)$ satisfies $R\left(x^{-1} y\right)$ $=R(y)$ for $\lambda^{r(x)}$-a.a. $y$.

Hence

$$
\int\left|R\left(x^{-1} y\right)-R(y)\right| d \lambda^{r(x)}(y)=0,
$$


so

$$
\int|R(y)-R(x y)| d \lambda^{d(x)}(y)=0
$$

so

$$
\begin{aligned}
0 & =\iiint|R(y)-R(x y)| d \lambda^{d(x)}(y) d \lambda_{u}(x) d \tilde{\lambda}(u) \\
& =\iiint|R(y)-R(x y)| d \lambda_{u}(x) d \lambda^{u}(y) d \tilde{\lambda}(u) \\
& =\iint|R(y)-R(x y)| d \lambda_{r(y)}(x) d \lambda(y) .
\end{aligned}
$$

Thus for $\lambda$-a.a. $y, R(y)=R(x y)$ for $\lambda_{r(y)}$-a.a. $x$. For such $y$,

$$
0=\int|R(y)-R(x y)| d \lambda_{r(y)}(x)=\int|R(y)-R(x)| d \lambda_{d(y)}(x) .
$$

Hence, if we define $\bar{R}(y)=\int R(z) d \lambda_{d(y)}(z)$, then $R(y)=\bar{R}(y)$ for $\lambda$-a.a. $y$. Also

$$
\bar{R}\left(x^{-1} y\right)=\int R(z) d \lambda_{d\left(x^{-1} y\right)}(z)=\int R(z) d \lambda_{d(y)}(z)=\bar{R}(y) .
$$

In particular, $\bar{R}(y)=\bar{R}\left(y^{-1} y\right)=\bar{R}(d(y))$. Thus, for $\lambda$-a.a. $y, P(y)=$ $P^{\prime}(y) \bar{R}(d(y))$. Since $0<\bar{R}(d(\underline{y}))<\infty$ for $\lambda$-a.a. $y$, if we define $\phi(u)=$ $\bar{R}(u)$ for $u \in U_{4}$ such that $0<\bar{R}(u)<\infty$ and $\phi(u)=1$ for all other $u \in U_{0}$ $\cap U_{0}^{\prime}$, then $\phi$ has the properties asserted.

REMARK 3.10. The proof of Theorem 3.9 shows that if a real-valued Borel function $R$ satisfies $R\left(x^{-1} y\right)=R(y) \lambda^{r(x)}$-a.e. for $\lambda$-a.a. $x$, then there is a Borel function $\phi$ on $U_{G}$ such that $R=\phi \circ d$ a.e. This fact identifies the invariant functions on $U_{G^{(2)}}$ as those of the form $\phi \circ r$.

Definition 3.11. Let $(G, C)$ be a measure groupoid. Let $\nu \in C$ and let $\mu \in \tilde{C}$ be a probability. The pair $(\nu, \mu)$ is called a Haar measure for $(G, C)$ if $\nu$ has an $r$-decomposition $\nu=\int \nu^{u} d \mu(u)$ with respect to $\mu$ such that for some i.r. $G_{0}$ of $G$,

$$
\begin{aligned}
& \text { for all } x \in G_{0} \text { and } f \geqslant 0 \text { Borel on } G \\
& \int f(y) d \nu^{r(x)}(y)=\int f(x y) d \nu^{d(x)}(y) .
\end{aligned}
$$

Corollary 3.13. For each probability $\mu \in \tilde{C} \exists \nu \in C$ such that $(\nu, \mu)$ is a Haar measure.

Proof. Let $\lambda \in C$ be a symmetric probability. Let $P, U_{0}$, and $u \mapsto \lambda^{u}$ be as provided by Theorem 3.9. Let $\nu^{u}(E)=\int 1_{E} P d \lambda^{u}$. Let

$$
\nu(E)=\int \nu^{u}(E) d \mu(u)=\iint 1_{E} P(d \mu / d \tilde{\lambda}) \circ r d \lambda .
$$


$(\nu, \mu)$ is a Haar measure with decomposition $\nu=\int \nu^{u} d \mu(u)$ satisfying (3.12) for $x \in G_{0}=G \mid U_{0}$.

COROllary 3.14. Let $(\nu, \mu)$ be a Haar measure such that $\nu$ has $r$-decomposition $\nu=\int \nu^{u} d \tilde{\lambda}(u)$ with respect to $\mu$. Let $U_{1} \subset U_{G}$ be a conull Borel set. Then there is a conull Borel set $U_{0} \subset U_{1}$ such that for $G_{0}=G \mid U_{0}$ we have

1. $u \in U_{0} \Rightarrow \nu^{u}\left(G-G_{0}\right)=0$.

2. $\forall$ Borel $f \geqslant 0$ on $G_{0}$ and $x \in G_{0}$,

$$
\int_{G_{0}} f(x y) d \nu^{d(x)}(y)=\int_{G_{0}} f(y) d \nu^{r(x)}(y) .
$$

3. $\exists$ a strict Borel homomorphism $\Delta: G_{0} \rightarrow \mathbf{R}_{+}^{*}$ such that $\Delta^{-1}=d \nu^{-1} / d \nu$.

Furthermore, if $\Delta^{\prime}=\left(d \nu^{-1} / d \nu\right)^{-1}$ is another homomorphism, then there is an i.r. $G_{2}$ of $G_{0}$ such that $\Delta^{\prime}\left|G_{2}=\Delta\right| G_{2}$. If $\left(\nu_{1}, \mu_{1}\right)$ is another Haar measure and $\Delta_{1}=\left(d \nu_{1}^{-1} / d \nu_{1}\right)^{-1}$, then there is a positive Borel function $\phi$ on $U_{G}$ such that a.e.

$$
\phi \circ d=\left(d \nu_{1} / d \nu\right)\left(d \mu / d \mu_{1}\right) \circ r \quad \text { and } \quad \frac{\Delta_{1}}{\Delta}=\frac{\phi \circ d}{\phi \circ r} \frac{\left(d \mu / d \mu_{1}\right) \circ r}{\left(d \mu / d \mu_{1}\right) \circ d} .
$$

Proof. Let $\nu=\int \nu^{u} d \mu(u)$ satisfy (3.12) for $x \in G_{3}=G \mid U_{3}, U_{3}$ conull Borel. Let $\lambda \in C$ be a symmetric probability. Let $p=d \nu / d \lambda$ and $\psi=d \mu / d \tilde{\lambda}$ be positive and Borel. $\lambda^{u}=E \mapsto \int 1_{E} p^{-1} \psi \circ r d \nu^{u}$ defines an $r$-decomposition $\lambda=\int \lambda^{u} d \tilde{\lambda}(u)$ by Theorem 2.1. Let $U_{4} \subset U_{1} \cap U_{3}$ be a conull Borel set such that $u \in U_{4} \Rightarrow \lambda^{u}\left(G \mid U_{4}\right)=1$. The proof of Theorem 3.9 shows that $\exists P$ and $U_{0} \subset U_{4}$ with properties 1,2 , and 3 of that theorem and such that $u \in U_{0} \Rightarrow P=p(\psi \circ r)^{-1} \lambda^{u}$-a.e.

$$
\begin{aligned}
\int f\left(y^{-1}\right) d \nu(y) & =\int f\left(y^{-1}\right) d \nu^{u}(y) d \mu(u) \\
& =\int f\left(y^{-1}\right) \hat{P}(y) d \lambda^{u}(y) \psi(u) d \tilde{\lambda}(u) \\
& =\int f(y) \frac{P\left(y^{-1}\right) \psi \circ r\left(y^{-1}\right)}{P(y) \psi \circ r(y)} P(y) \psi \circ r(y) d \lambda(y) \\
& =\int f(y) \frac{P\left(y^{-1}\right)}{P(y)} \frac{\psi(d(y))}{\psi(r(y))} d \nu(y) .
\end{aligned}
$$

Hence

$$
\left(\frac{d \nu^{-1}}{d \nu}\right)^{-1}=\Delta=y \mapsto \frac{P(y)}{P\left(y^{-1}\right)} \frac{\psi(r(y))}{\psi(d(y))}
$$

is a strict homomorphism.

If $\Delta^{\prime}=\left(d \nu^{-1} / d \nu\right)^{-1}$ is strict on another i.r., then $\Delta=\Delta^{\prime}$ on a conull 
multiplicative Borel set. Applying Theorem 2.10, we obtain an i.r. on which $\Delta$. and $\Delta^{\prime}$ agree.

Now if $\left(\nu_{1}, \mu_{1}\right)$ is another Haar measure, let $p_{1}=d \nu_{1} / d \lambda, \psi_{1}=d \mu_{1} / d \tilde{\lambda}$, $\lambda_{1}^{u}=E \mapsto \int 1_{E} p_{1}^{-1} \psi_{1} \circ r d \nu_{1}^{u}, P_{1}$ the function corresponding to $P$ above, $U_{01}$ corresponding to $U_{0}$. Let $U_{5} \subset U_{0} \cap U_{01}$ be a conull Borel set such that $u \in U_{5} \Rightarrow \lambda^{u}\left(G \mid U_{5}\right)=1$ and $\lambda_{1}^{u}=\lambda^{u}$. Then by the theorem there is a positive Borel $\phi$ such that $P_{1} / P=\phi \circ d$ a.e.

$$
\begin{aligned}
\frac{P_{1}}{P} & =\frac{p_{1}\left(\psi_{1} \circ r\right)^{-1}}{p(\psi \circ r)^{-1}}=\frac{d \nu_{1} / d \lambda}{d \nu / d \lambda}\left(\frac{d \mu_{1} / d \tilde{\lambda}}{d \mu / d \tilde{\lambda}}\right)^{-1} \circ r \\
& =\left(d \nu_{1} / d \nu\right)\left(d \mu / d \mu_{1}\right) \circ r \text { a.e. }
\end{aligned}
$$

and

$$
\begin{aligned}
\frac{\Delta_{1}(y)}{\Delta(y)} & =\frac{\left(P_{1}(y) / P_{1}\left(y^{-1}\right)\right)\left(\psi_{1} \circ r(y) / \psi_{1} \circ d(y)\right)}{\left(P(y) / P\left(y^{-1}\right)\right)(\psi \circ r(y) / \psi \circ d(y))} \\
& =\left(\frac{\phi \circ d(y)}{\phi \circ r(y)}\right)\left(\frac{d \mu_{1}}{d \mu}\right) \circ r\left(\frac{d \mu_{1}}{d \mu}\right)^{-1} \circ d \text { a.e. }
\end{aligned}
$$

Definition 3.15. A Borel function $\Delta=\left(d \nu^{-1} / d \nu\right)^{-1}$ which is a homomorphism is called the modular homomorphism of $(\nu, \mu)$.

Strictly speaking, $\Delta$ is determined only up to a null Borel set. This causes no difficulty. The last equation of Corollary 3.14 shows that replacing $(\nu, \mu)$ by $\left(\nu_{1}, \mu_{1}\right)$ replaces $\Delta$ by a similar homomorphism [13, Definition 6.4].

EXAMPLE 3.16. For the groupoid $(S \times g,[\mu \times h])$ of Example 2.5, $(\mu \times$ $h, \mu)$ is a Haar measure. Then $\Delta^{-1}$ is a Radon-Nikodým derivative of $(\mu \times h)^{-1}$ with respect to $(\mu \times h)$. Let $\delta=\left(d h^{-1} / d h\right)^{-1}$ be the modular homomorphism for the left Haar measure $h$ of g. Then for a.a. $x \in \mathrm{g}$,

$$
\delta(x) \int 1_{E}(s x) \Delta(s, x)^{-1} d \mu(s)=\int 1_{E}(s) d \mu(s),
$$

so that for a.a. $x, s \mapsto \Delta(s, x) / \delta(x)$ is a Radon-Nikodým derivative of $E \mapsto \int 1_{E}(s x) d \mu(s)$ with respect to $\mu$. See [11, p. 198]. See also Remark 4.12 concerning Haar measures constructed in special cases.

REMARK 3.17. If $\left(\nu_{1}, \mu_{1}\right)$ is a Haar measure with $r$-decomposition $\nu_{1}=$ $\int \nu^{u} d \mu_{1}(u)$ and $\mu \sim \mu_{1}$ is another probability, then $\nu=\int \nu^{u} d \mu(u)$ is such that $(\nu, \mu)$ is a Haar measure. This follows from Corollary 3.14. Thus if $\lambda$ and $P$ are as in Theorem 3.9, the most general Haar measure is obtained as follows: choose positive Borel functions $\phi, \psi$ on $U_{G}$ such that $\int \psi d \tilde{\lambda}=1$. Define $\nu(E)=\int_{E} P \phi \circ d \psi \circ r d \lambda$ and $\mu(F)=\int_{F} \psi d \tilde{\lambda} .(\nu, \mu)$ is a Haar measure. From this one sees that if $G$ is ergodic, $\nu$ determines $\mu$. Indeed, if also $\left(\nu, \mu^{\prime}\right)$ is a Haar measure, defined by $\phi^{\prime}$ and $\psi^{\prime}$, then $(\phi \circ d)(\psi \circ r)=\left(\phi^{\prime} \circ d\right)\left(\psi^{\prime} \circ r\right)$ 
a.e. For a.a. $u, \phi \circ d / \phi^{\prime} \circ d$ is $\lambda^{u}$-essentially constant, equal to $\int \phi / \phi^{\prime} d\left(d_{*} \lambda^{u}\right)$. Since for a.a. $x, \int \phi / \phi^{\prime} d\left(d_{*} \lambda^{d(x)}\right)=\int \phi / \phi^{\prime} d\left(d_{*} \lambda^{r(x)}\right)$, by ergodicity $\phi / \phi^{\prime}$ is essentially constant. Thus $\psi=\psi^{\prime}$ a.e. and $\mu=\mu^{\prime}$, because $\mu$ and $\mu^{\prime}$ are probabilities.

4. Further decomposition of the Haar measure. Let $(G, C)$ be a measure groupoid, $\lambda \in C$ a symmetric probability. Let $\delta=(r, d)(G) \subset U_{G} \times U_{G}$ and $C^{\prime}=(r, d)_{*} C$ as in Example 2.8. Here we adopt Ramsay's right invariance notation (e.g. $\lambda_{r(x)} \cdot x=E \mapsto \int 1_{E}(y x) d \lambda_{r(x)}(y)$ ) and state his Lemma 6.6 of [14].

LEMMA 4.1. Let $(G,[\lambda])$ be a measurable groupoid with $\lambda(G)=1$ and $\lambda=\lambda^{-1}$. Let $\mathcal{E}=(r, d)(G)$ be the associated equivalence relation on $U=U_{G}$ and set $\lambda^{\prime}=(r, d)_{*}(\lambda)$. Let $\lambda^{\prime}=\int \lambda_{u}^{\prime} d \tilde{\lambda}(u)$ be a decomposition of $\lambda^{\prime}$ relative to $d$; let $\lambda=\int \lambda_{u, v} d \lambda^{\prime}(u, v)$ be a decomposition of $\lambda$ relative to $(r, d)$; for $u \in U$ let $\lambda_{u}^{\prime \prime}=r_{*}\left(\lambda_{u}^{\prime}\right)$ and set $\lambda_{u}=\int \lambda_{v, u} d \lambda_{u}^{\prime \prime}(v)$. Then $\lambda=\int \lambda_{u} d \tilde{\lambda}(u)$ is a decomposition of $\lambda$ relative to $d$, and there is a conull Borel set $U_{0} \subset U$ such that if $G_{0}=G \mid U_{0}$ and $\varepsilon_{0}=\mathcal{E} \mid U_{0}$, then:

1. $u \in U_{0}$ implies $\lambda_{u}^{\prime}\left(\mathcal{G}_{0}\right)=\lambda_{u}^{\prime}([u] \times\{u\})=1($ recall $[u]=\{v: v \sim u\})$;

2. $(u, v) \in \mathcal{E}_{0}$ implies $\lambda_{u}^{\prime} \cdot(u, v) \sim \lambda_{v}^{\prime}$;

3. $u \in U_{0}$ implies $\lambda_{u}\left(G_{0}\right)=\lambda_{u}\left(d^{-1}(u)\right)=1$;

4. $x \in G_{0}$ implies $\lambda_{r(x)} \cdot x \sim \lambda_{d(x)}$.

Also, the $\lambda_{u, v}$ may be chosen so that $\lambda_{u, v}=\left(\lambda_{v, u}\right)^{-1}$, and if $\lambda^{u}=\left(\lambda_{u}\right)^{-1}$, then $\int \lambda^{u} d \tilde{\lambda}(u)$ is a left quasi-invariant decompositon of $\lambda$.

Ramsay also shows that if $\lambda$ is chosen properly, the measures $\lambda_{u, v}$ satisfy the quasi-invariance condition $\lambda_{u, r(x)} \cdot x \sim \lambda_{u, d(x)}$ on an i.r. [14, Theorem 6.8], and concludes that a.a. $G \mid\{u\}$ are the Borel groups of locally compact groups with Haar measure class $\left[\lambda_{u, u}\right][14$, Theorem 6.9, p. 95]. He asks whether there is a measure $\nu$ in $C$ with decomposition over $\delta$ such that a.e. $\nu_{u, u}$ actually is the Haar measure on $G \mid\{u\}$. We will show that our Haar measure on $G$ found in $\$ 3$ answers this question in the affirmative.

The first step is to convert Theorem 3.9 to right invariance. $Q$ is $y \mapsto$ $P\left(y^{-1}\right)$.

THEOREM 4.2. Let $(G, C)$ be an analytic groupoid with invariant measure class and $\lambda \in C$ a symmetric probability. There is a conull Borel set $U_{0} \subset U_{G}$ and a Borel function $Q: G \mid U_{0} \rightarrow \mathbf{R}_{+}-\{0\}$ such that

1. $\lambda$ has a d-decomposition $\lambda=\int \lambda_{u} d \tilde{\lambda}(u)$ on $G_{0}=G \mid U_{0}$ such that $\lambda_{u}\left(G_{0}\right)=$ 1 for all $u \in U_{0}$..

2. For all $f: G_{0} \rightarrow \mathbf{R}_{+}$Borel and all $x \in G_{0}$,

$$
\int f(y x) Q(y) d \lambda_{r(x)}(y)=\int f(y) Q(y) d \lambda_{d(x)}(y) .
$$


3. $y \mapsto Q\left(y^{-1}\right) / Q(y)$ is a strict homomorphism of $G_{0}$ into $\mathbf{R}_{+}^{*}$.

By considering the special case $\left(\mathcal{E}, C^{\prime}\right)$ we obtain

COROLLARY 4.3. There is a conull Borel set $U_{0}^{\prime} \subset U_{G}$ and a Borel function q: $\mathcal{E} \mid U_{0}^{\prime} \rightarrow \mathbf{R}_{+}-\{0\}$ such that

1. $\lambda^{\prime}$ has a d-decomposition $\lambda^{\prime}=\int \lambda_{u}^{\prime} d \tilde{\lambda}(u)$ on $\mathcal{E}_{0}=\mathcal{E} \mid U_{0}^{\prime}$ such that $\lambda_{u}^{\prime}\left(\mathcal{E}_{0}\right)$ $=1$ for all $u \in U_{0}^{\prime}$.

2. For all $f: \mathcal{E}_{0} \rightarrow \mathbf{R}_{+}$Borel and all $(u, v) \in \mathcal{E}_{0}$,

$$
\begin{aligned}
& \int f\left(\left(u_{1}, v_{1}\right)(u, v)\right) q\left(u_{1}, v_{1}\right) d \lambda_{u}^{\prime}\left(u_{1}, v_{1}\right) \\
& =\int f\left(u_{1}, v_{1}\right) q\left(u_{1}, v_{1}\right) d \lambda_{v}\left(u_{1}, v_{1}\right) .
\end{aligned}
$$

3. $(u, v) \mapsto q(v, u) / q(u, v)$ is a strict homomorphism of $\mathcal{E}_{0}$ into $\mathbf{R}_{+}^{*}$.

Let $U_{0}^{\prime \prime}=\left\{u \in U_{0} \cap U_{0}^{\prime}: \lambda_{u}=\int \lambda_{v, u} d \lambda_{u}^{\prime \prime}(v)\right\}$, where $\lambda_{u}$ is as in Theorem 4.2, $\lambda_{u}^{\prime \prime}=r_{*}\left(\lambda_{u}^{\prime}\right), \lambda_{u}^{\prime}$ is as in Corollary 4.3, and $\lambda_{v, u}$ as in Lemma 4.1. $U_{0}^{\prime \prime}$ is conull and Borel since $\mathfrak{B}(G)$ is countably generated.

Let

$$
\begin{aligned}
& U_{1}=\left\{u \in U_{0}^{\prime \prime}: \lambda_{u}\left(G \mid U_{0}^{\prime \prime}\right)=\lambda_{u}^{\prime}\left(E \mid U_{0}^{\prime \prime}\right)=1\right\}, \\
& U_{2}=\left\{u \in U_{1}: \lambda_{u}\left(G \mid U_{1}\right)=\lambda_{u}^{\prime}\left(E \mid U_{1}\right)=1\right\}, \\
& \vdots \\
& U_{n}=\left\{u \in U_{n-1}: \lambda_{u}\left(G \mid U_{n-1}\right)=\lambda_{u}^{\prime}\left(E \mid U_{n-1}\right)=1\right\} \\
& \vdots \\
& \bigcap_{n=1}^{\infty} U_{n} \text { is a conull Borel set and } \\
& u \in \cap U_{n} \Rightarrow \lambda_{u}\left(G \mid \cap U_{n}\right)=\lim \lambda_{u}\left(G \mid U_{n}\right)=1 \\
& \quad=\lim \lambda_{u}^{\prime}\left(\mathscr{E} \mid U_{n}\right)=\lambda_{u}^{\prime}\left(\mathscr{E} \mid \cap U_{n}\right),
\end{aligned}
$$

so we may assume that given $(G, C)$ and related $\left(\mathscr{E}, C^{\prime}\right)$ and $\lambda, U_{0}$ of Theorem 4.2 and $U_{0}^{\prime}$ of Corollary 4.3 are the same, and that $\lambda_{u}=\int \lambda_{v, u} d \lambda_{u}^{\prime \prime}(v)$ for all $u \in U_{0}$.

Let

$$
\begin{aligned}
& \delta=y \mapsto \frac{Q(y) q(d(y), r(y))}{Q\left(y^{-1}\right) q(r(y), d(y))} \quad \text { and } \\
& \square=y \mapsto Q(y) / q(r(y), d(y)) .
\end{aligned}
$$

Then $\delta: G_{0} \rightarrow \mathbf{R}_{+}^{*}$ is a strict Borel homomorphism and $\delta=y \mapsto$ $\square(y) / \square\left(y^{-1}\right)$. 
THEOREM 4.4. On $G_{0}=G \mid U_{0}$, the integral $f \mapsto \int f(y) Q(y) d \lambda(y)$ has $(r, d)$-decomposition

$$
\int_{\mathscr{E}_{0}} \int_{G_{0}} f(y) d v_{u, v}(y) q(u, v) d \lambda^{\prime}(u, v)
$$

with respect to $\lambda^{\prime}$ on $\mathscr{E}_{0}=\mathcal{E} \mid U_{0}$ such that

1. For all $(u, v) \in \mathcal{E}_{0}, \nu_{u, v}$ is $\sigma$-finite.

2. For all $f \geqslant 0$ Borel on $G_{0},(u, v) \mapsto \int_{G_{0}} f(y) d v_{u, v}(y)$ is an extended real-valued Borel function.

3. For all $f \geq 0$ Borel on $G_{0}$,

$$
\int_{G_{0}} f(y x) d v_{u, r(x)}(y)=\int_{G_{0}} f(y) d v_{u, d(x)}(y)
$$

whenever $(u, r(x))$ and $(u, d(x))$ are in $\mathcal{E}_{0}$.

4. For all $f \geqslant 0$ Borel on $G_{0}$,

$$
\delta(x) \int_{G_{0}} f(x y) d v_{d(x), v}(y)=\int_{G_{0}} f(y) d v_{r(x), v}(y)
$$

whenever $(d(x), v)$ and $(r(x), v)$ are in $\mathcal{E}_{0}$.

Thus $\nu_{u, u}$ is a right Haar measure on $G \mid\{u\}$ for all $u \in U_{0}$ and $\delta^{-1} \mid(G \mid\{u\})$ is its modular function.

Proof. Without loss of generality, we may assume $U_{0}=U_{G}$ and work on $G$ and $\mathcal{E}$. Write $U$ for $U_{G}=U_{\mathcal{E}}$. Let $\phi$ be a nonnegative Borel function on $U$.

$$
\begin{aligned}
\int_{U} \phi(u) q(u, d(z)) d \lambda_{d(z)}^{\prime \prime}(u)=\int_{\mathscr{E}} \phi(u) q(u, v) d \lambda_{d(z)}^{\prime}(u, v) \\
=\int_{\mathscr{E}} \phi(u) q(u, v) d \lambda_{r(z)}^{\prime}(u, v)=\int_{U} \phi(u) q(u, r(z)) d \lambda_{r(z)}^{\prime \prime}(u), \\
\int_{U} \int_{G} f(y z) \square(y) q(r(y), d(y)) \phi(r(y)) d \lambda_{u, r(z)}(y) d \lambda_{r(z)}^{\prime \prime}(u) \\
=\int_{U} \int_{G} f(y z) Q(y) \phi(r(y)) d \lambda_{u, r(z)}(y) d \lambda_{r(z)}^{\prime \prime}(u) \\
=\int_{G} f(y z) Q(y) \phi(r(y)) d \lambda_{r(z)}(y) \\
=\int_{G} f(y) Q(y) \phi(r(y)) d \lambda_{d(z)}(y) \\
=\int_{U} \int_{G} f(y) Q(y) \phi(r(y)) d \lambda_{u, d(z)}(y) d \lambda_{d(z)}^{\prime \prime}(u) \\
=\int_{U} \int_{G} f(y) \square(y) q(r(y), d(y)) \phi(r(y)) d \lambda_{u, d(z)}(y) d \lambda_{d(z)}^{\prime \prime}(u) .
\end{aligned}
$$

From these equations we obtain 


$$
\begin{aligned}
\int_{U} \phi(u)\left[\int_{G} f(y z) \square(y) d \lambda_{u, r(z)}(y)\right] q(u, r(z)) d \lambda_{r(z)}^{\prime \prime}(u) \\
\quad=\int_{U} \phi(u)\left[\int_{G} f(y) \square(y) d \lambda_{u, d(z)}(y)\right] q(u, d(z)) d \lambda_{r(z)}^{\prime \prime}(u) \\
=\int_{U} \phi(u)\left[\int_{G} f(y) \square(y) d \lambda_{u, d(z)}(y)\right] q(u, r(z)) d \lambda_{r(z)}^{\prime \prime}(u)
\end{aligned}
$$

by substituting $u \mapsto \phi(u)\left[\int_{G} f(y) \square(y) d \lambda_{u, d(z)}(y)\right]$ for $\phi$ in (4.5). Since $\mathscr{B}(G)$ is countably generated,

$$
\begin{aligned}
\{u \in U: \text { for all Borel } f \geqslant 0, & \int_{G} f(y z) \square(y) d \lambda_{u, r(z)}(y) \\
& \left.=\int_{G} f(y) \square(y) d \lambda_{u, d(z)}(y)\right\} \text { is } \lambda_{r(z)}^{\prime \prime} \text {-conull. }
\end{aligned}
$$

Let

$$
\begin{aligned}
A_{v}= & \left\{(u, x) \in[v] \times d^{-1}(\{v\}): \text { for all Borel } f \geqslant 0,\right. \\
& \left.\int f(y x) \square(y) d \lambda_{u, r(x)}(y)=\int f(y) \square(y) d \lambda_{u, d(x)}(y)\right\}
\end{aligned}
$$

(cf. proof of Ramsay's Lemma 6.7 of [14]). Since both integrals are Borel functions of $(u, x)$ and $\mathscr{B}(G)$ is countably generated, $A_{v}$ is Borel for all $v \in U$. If $d(x)=v$, then $\left\{u:(u, x) \in A_{v}\right\}$ is $\left[\lambda_{r(x)}^{\prime \prime}\right]=\left[\lambda_{v}^{\prime \prime}\right]$-conull by (4.6). By Fubini's Theorem, $B_{v}=\left\{u: \lambda_{v}\left(\left\{x:(u, x) \in A_{v}\right\}\right)\right\}=1$ is $\lambda_{v}^{\prime \prime}$-conull for all $v \in U$.

Define the measures $\nu_{u, v}$ by

$$
\int f(y) d v_{u, v}(y)=\iint f(y x) \square(y) d \lambda_{u, r}(x)(y) d \lambda_{v}(x)
$$

For $u \in B_{v}$,

$$
\begin{aligned}
\int f(y) d \nu_{u, v}(y) & =\iint f(y) \square(y) d \lambda_{u, d}(x)(y) d \lambda_{v}(x) \\
& =\iint f(y) \square(y) d \lambda_{u, v}(y) d \lambda_{v}(x) \\
& =\int f(y) \square(y) d \lambda_{u, v}(y) .
\end{aligned}
$$

By Lemma 2.8 of [14] $(u, v) \mapsto \int f(y) d v_{u, v}(y)$ is Borel. Since $B_{v}$ is $\left[\lambda_{v}^{\prime \prime}\right]$ conull,

$$
\begin{aligned}
\int f(y) & d \nu_{u, v}(y) q(u, v) d \lambda_{v}^{\prime \prime}(u) \\
& =\iint f(y) \square(y) d \lambda_{u, v}(y) q(u, v) d \lambda_{v}^{\prime \prime}(u) \\
& =\int f(y) Q(y) d \lambda_{v}(y)
\end{aligned}
$$


so the measures $q(u, v) \nu_{u, v}$ define an $(r, d)$-decomposition of $f \mapsto$ $\int f(y) Q(y) d \lambda(y)$ with respect to $\lambda^{\prime}$ satisfying the second condition of the theorem.

There is at least one $u$ in $B_{v}$. If $u^{\prime} \in[v]=[u]$ also, $\exists z$ such that $d(z)=u$, $r(z)=u^{\prime}$. Let $\lambda^{w}=E \mapsto \lambda_{w}\left(E^{-1}\right)$. We have

$$
\begin{aligned}
\int f(w) d\left(d_{*} \lambda^{v}\right)(w) & =\int f(d(y)) d \lambda^{v}(y)=\int f(r(y)) d \lambda_{v}(y) \\
& =\iint f(r(y)) d \lambda_{w, v}(y) d \lambda_{v}^{\prime \prime}(w)=\int f(w) d \lambda_{v}^{\prime \prime}(w),
\end{aligned}
$$

so that $d_{*} \lambda^{0}=\lambda_{v}^{\prime \prime}$. By (4.6),

$$
\begin{array}{r}
\left\{w \in U: \text { for all Borel } f \geqslant 0, \int_{G} f(y z) \square(y) d \lambda_{w, r(z)}(y)\right. \\
\left.=\int_{G} f(y) \square(y) d \lambda_{w, v}(y)\right\}
\end{array}
$$

is $\left[\lambda_{r(z)}^{\prime \prime}\right]=\left[\lambda_{d(z)}^{\prime \prime}\right]=\left[\lambda_{v}^{\prime \prime}\right]$-conull, so

$$
\begin{array}{r}
\left\{x \in r^{-1}\{v\}: \text { for all Borel } f \geqslant 0, \int_{G} f(y z) \square(y) d \lambda_{d(x), r(z)}(y)\right. \\
\left.=\int_{G} f(y) \square(y) d \lambda_{d(x), d(z)}(y)\right\}
\end{array}
$$

is $\lambda^{v}$-conull.

Thus

$$
\begin{aligned}
\delta(z)^{-1} \int f(y) d \nu_{u^{\prime}, v}(y) \\
=\iint f(y z) \square(y) \delta(z)^{-1} d \lambda_{r(z), r(x)}(y) d \lambda_{v}(x) \\
=\iint f\left(y x^{-1}\right) \square(y) \delta(z)^{-1} d \lambda_{r(z), d(x)}(y) d \lambda^{v}(x) \\
=\iint f\left(y^{-1} x^{-1}\right) \square\left(y^{-1}\right) \delta(z)^{-1} d \lambda_{d(x), r(z)}(y) d \lambda^{v}(x) \\
=\iint f\left((x y)^{-1}\right) \square(y) \delta(y)^{-1} \delta(z)^{-1} d \lambda_{d(x), r(z)}(y) d \lambda^{v}(x) \\
=\iint f\left((x y)^{-1}\right) \square(y) \delta(y z)^{-1} d \lambda_{d(x), r(z)}(y) d \lambda^{v}(x) \\
=\iint f\left(\left(x y z^{-1}\right)^{-1}\right) \square(y) \delta(y)^{-1} d \lambda_{d(x), d(z)}(y) d \lambda^{v}(x)
\end{aligned}
$$




$$
\begin{aligned}
& =\iint f\left(\left(x y^{-1} z^{-1}\right)^{-1}\right) \square\left(y^{-1}\right) \delta(y) d \lambda_{d(z), d(x)}(y) d \lambda^{v}(x) \\
& =\iint f\left(\left(x^{-1} y^{-1} z^{-1}\right)^{-1}\right) \square\left(y^{-1}\right) \delta(y) d \lambda_{d(z), r(x)}(y) d \lambda_{v}(x) \\
& =\iint f(z y x) \square(y) d \lambda_{d(z), r(x)}(y) d \lambda_{v}(x) \\
& \left.\quad \text { (recall that } \square\left(y^{-1}\right) \delta(y)=\square(y)\right) \\
& =\int f(z y) d \nu_{u, v}(y) .
\end{aligned}
$$

Thus $\nu_{u^{\prime}, v}$ is $\sigma$-finite whenever $\nu_{u, v}$ is. Since $u \in B_{v}$,

$$
\nu_{u, v}=F \mapsto \int_{F} \square(y) d_{u, v}(y)
$$

is $\sigma$-finite, so all $\nu_{u^{\prime}, v}$ for $u^{\prime} \in[v]$ are $\sigma$-finite. This is the first condition of the theorem.

$$
\text { If } \begin{aligned}
r\left(z_{0}\right)= & u^{\prime \prime} \text { and } d\left(z_{0}\right)=u^{\prime} \text {, then } r\left(z_{0} z\right)=u^{\prime \prime} \text { and } d\left(z_{0} z\right)=u \text {, so } \\
& \delta\left(z_{0} z\right)^{-1} \int f(y) d v_{r\left(z_{0}\right), v}(y)=\int f\left(z_{0} z y\right) d v_{d(z), v}(y)
\end{aligned}
$$

and

$$
\delta(z)^{-1} \int f\left(z_{0} y\right) d v_{d\left(z_{0}\right), v}(y)=\int f\left(z_{0} z y\right) d v_{d(z), v}(y)
$$

hence

$$
\delta\left(z_{0}\right)^{-1} \int f(y) d \nu_{r\left(z_{0}\right), v}(y)=\int f\left(z_{0} y\right) d v_{d\left(z_{0}\right), v}(y) .
$$

This is the fourth condition of the theorem.

If $d(w)=v$, then

$$
\begin{aligned}
\int f(y w) d v_{u, r(w)}(y) & =\iint f(y x w) \square(y) d \lambda_{u, r(x)}(y) d \lambda_{r(w)}(x) \\
& =\iint f(y(x w)) \square(y) d \lambda_{u, r(x w)}(y) d \lambda_{r(w)}(x) \\
& =\iint f(y x) \square(y) d \lambda_{u, r(x)}(y) d\left(\lambda_{r(w)} \cdot w\right)(x) .
\end{aligned}
$$

Since $u \in B_{v}=B_{d(w)}$, for $\left[\lambda_{v}\right]=\left[\lambda_{r(w)} \cdot w\right]$-a.a. $x$, we have

$$
\begin{aligned}
\int f(y x) \square(y) d \lambda_{u, r(x)}(y) & =\int f(y) \square(y) d \lambda_{u, d(x)}(y) \\
& =\int f(y) \square(y) d \lambda_{u, v}(y),
\end{aligned}
$$

which is a constant $c$ independent of $x$, so that 


$$
\begin{aligned}
\int f(y w) d \nu_{u, r(w)}(y) & =\int c d\left(\lambda_{r(w)} \cdot w\right)(x)=c=\int c d \lambda_{d(w)}(x) \\
& =\iint f(y x) \square(y) d \lambda_{u, r(x)}(y) d \lambda_{d(w)}(x) \\
& =\int f(y) d \nu_{u, d(w)}(y) .
\end{aligned}
$$

The third condition, general right invariance, now follows from this and the general left invariance (4.8):

$$
\begin{gathered}
\delta(z)^{-1} \int f(y w) d v_{u^{\prime}, r(w)}(y)=\delta(z)^{-1} \int f(y w) d v_{r(z), r(w)}(y) \\
=\int f(z y w) d v_{d(z), r(w)}(y)=\int f(z y w) d v_{u, r(w)}(y) \\
=\int f(z y) d v_{u, d(w)}(y)=\int f(z y) d v_{d(z), d(w)}(y) \\
=\delta(z)^{-1} \int f(y) d v_{r(z), d(w)}(y)=\delta(z)^{-1} \int f(y) d v_{u^{\prime}, d(w)}(y)
\end{gathered}
$$

Corollary 4.9. If $(G, C)$ is ergodic, then either $\tilde{C}$-a.a. $G \mid\{u\}$ are unimodular or $\tilde{C}$-a.a. $G \mid\{u\}$ are nonunimodular.

Proof. Let $F=\left\{x \in G_{0}: \delta(x) \neq 1\right\}$. $F$ is Borel. $G \mid\{u\}$ is unimodular iff $\lambda_{u, u}(F)=0$.

$$
\begin{aligned}
\lambda_{d(x), d(x)}(F)=0 & \text { iff } x^{-1} \cdot\left(\lambda_{r(x), r(x)} \cdot x\right)(F)=0 \\
& \text { iff } \lambda_{r(x), r(x)}\left(x(F \cap G \mid\{d(x)\}) x^{-1}\right)=0 \\
& \text { iff } \lambda_{r(x), r(x)}(F)=0
\end{aligned}
$$

since $\delta$ is a strict homomorphism of $G_{0}$ into $\mathbf{R}_{+}^{*}$. Thus if $U_{1}=\left\{u \in U_{0}\right.$ : $\left.\lambda_{u, u}(F)=0\right\}$ then $1_{U_{1}} \circ d=1_{U_{1}} \circ r$ on $G_{0}$, so $U_{1}$ is either null or conull.

REMARK 4.10. If $G$ is a principal groupoid, Theorem 4.4 contains no information not in Theorem 4.2.

REMARK 4.11. Theorem 4.4 can be translated easily to a result about left invariance. We omit an explicit statement.

REMARK 4.12. Westman [17, p. 622] has proved the existence when $G$ is transitive of a measure with invariant decomposition like that of Theorem 4.4. He uses such a measure to develop a theory of groupoid algebra, first in the transitive case [17], then the principal case [18], and then the general case [19]. In the nontransitive situations [18], [19] his investigation requires the assumption that such a measure exists. Our Theorem 4.4 may thus be regarded as a proof that every measure groupoid in the sense of Mackey and Ramsay has an i.r. which is a groupoid of the type considered by Westman. Seda [16], also, has studied Haar measure for transitive groupoids. 
If $g$ acts on $S$ as in Examples 2.5 and 3.16, but the action is not free, then the Haar measure on the associated principal groupoid as in Corollary 4.3 is not easy to construct explicitly. This has been accomplished by Samuelides and Sauvageot [15].

5. The case of finite Haar measure. The existence of an analogue for measure groupoids of the Haar measure for locally compact groups reinforces our belief that groupoids possess many group-like properties. Sometimes some property of separable locally compact groups must be reformulated in order to extend it to measure groupoids. For example, consider group representations. If one expects "strongly continuous homomorphisms" of a groupoid into the unitary group of a Hilbert space, he must be disappointed because there is as yet no topology for measure groupoids. However, if $g$ is a 1.c.s.c. group, the strongly continuous representations of $g$ in separable Hilbert space coincide with the strongly Borel ones; strongly Borel homomorphisms into the unitary group are exactly what is meant by a representation of a groupoid in separable Hilbert space. Reformulation of topological properties of groups in terms of algebraic and measure-theoretic characteristics is one aspect of what Mackey has called "the virtual group point of view." One such property we have specifically in mind is compactness; a l.c. group is compact iff its Haar measure is finite.

The theory of virtual groups is more concrete than simply a point of view, however. Using a concept of homomorphisms between measure groupoids and a measure-theoretic generalization of natural equivalence of functors, one can define what is meant for two ergodic groupoids to be similar; this defines an equivalence relation on any set of ergodic groupoids. Similar groupoids are said to define the same virtual group.

For an extensive discussion of similarity and its consequences, the reader is referred to Ramsay's paper [13]. However, we must make two comments. First, the term virtual group in [13] is used to describe what we call an ergodic groupoid. Second, there are two concepts of homomorphism between ergodic groupoids; consequently, there are two concepts of similarity. Let $\left(G_{1}, C_{1}\right)$ and $\left(G_{2}, C_{2}\right)$ be ergodic groupoids, $\phi$ a strict Borel homomorphism from the Borel groupoid $G_{1}$ into the Borel groupoid $G_{2}$. In Mackey's definition, for $\phi$ to be a homomorphism $\tilde{\phi}^{-1}(N)$ must be $\tilde{C}_{1}$-null whenever $N \in \mathscr{B}\left(U_{G_{2}}\right)$ is $\tilde{C}_{2}$-null and contained in the union of the null equivalence classes [10, p. 1186]. In Ramsay's definition, [13, p. 286], $\tilde{\phi}^{-1}(N)$ need not be null if $[N]=d\left(r^{-1}(N)\right)$ is conull.

Let $(G, C)$ be a measure groupoid. One says that a Haar measure $(\nu, \mu)$ of $(G, C)$ is finite if $\nu(G)$ is finite. We expect ergodic groupoids possessing a finite Haar measure to be special, and they are. They are essentially transitive; and in the decomposition described by Theorem 4.4, a.a. $\nu_{u, u}$ are finite. 
Put another way, they are those essentially transitive groupoids similar to compact groups. The theorem is valid with either definition of similarity.

THEOREM 5.1. Let $(G, C)$ be an ergodic groupoid. The following are equivalent:

1. $(G, C)$ is similar to a compact group.

2. $(G, C)$ has a finite Haar measure.

3. $(G, C)$ is essentially transitive and for some symmetric probability $\lambda \in C$ and some Haar measure $(\nu, \tilde{\lambda})$, in the $(r, d)$-decomposition of $\nu$ with respect to $\lambda^{\prime}$ in Theorem 4.4, a.a. $\nu_{u, u}$ are finite.

4. There is a symmetric probability $\lambda \in C$ such that $(\lambda, \tilde{\lambda})$ is a Haar measure.

Proof. We use certain facts about similarity; these facts hold for either definition. $G$ is similar to its inessential reductions [13, Theorem 6.16]. $G$ is essentially transitive iff $G$ is similar to a group, which is uniquely determined. If this is the case, there is $u_{0} \in U_{G}$ such that $\left[\left\{u_{0}\right\}\right]$ is $\tilde{C}$-conull and $G$ is similar to the group whose underlying Borel group is $G \mid\left\{u_{0}\right\}[13$, Theorem 6.19 and remark following Theorem 6.20, p. 293]. The similarity of $G$ and $G \mid\left\{u_{0}\right\}$ is effected by finding an i.r. $G \mid U_{1}$ and a Borel cross-section $\gamma$ : $U_{1} \rightarrow r^{-1}\left(\left\{u_{0}\right\}\right)$ of $d$ on $U_{1}$ such that $u_{0} \in U_{1} \subset\left[\left\{u_{0}\right\}\right]$ and $\gamma\left(u_{0}\right)=u_{0}[13$, proof of Theorem 6.17]; then one homomorphism $\psi$ of the similarity is defined on $G \mid U_{1}$ by $\psi(x)=\gamma(r(x)) x \gamma(d(x))^{-1}$, and the other is the inclusion $G \mid\left\{u_{0}\right\} \rightarrow G$. The invariant measure class on $G \mid\left\{u_{0}\right\}$ is $\psi_{*}(C)$ and it contains the Haar measure of $G \mid\left\{u_{0}\right\}$.

$(1 \Rightarrow 2)$ In view of the definition, it is enough to prove that $G$ has an i.r. with finite Haar measure. Thus we may assume that $U_{G}$ itself has a Borel cross-section $\gamma: U_{G} \rightarrow r^{-1}\left(\left\{u_{0}\right\}\right)$ of $d$ such that $\gamma\left(u_{0}\right)=u_{0}$. Let $\lambda \in C$ be a symmetric probability, $U_{2} \subset U_{G}$ a conull Borel set such that $\lambda$ has decomposition $\lambda=\int \lambda^{u} d \tilde{\lambda}(u)$ over $r$ on $G \mid U_{2}$ satisfying $\lambda^{u}(G)=\lambda^{u}\left(G \mid U_{2}\right)=1$ for all $u \in U_{2}$ and $x \lambda^{d(x)} \sim \lambda^{r(x)} \forall x \in G \mid U_{2}$.

Let $u_{1} \in U_{2}$ and let $\gamma_{1}(u)=\gamma\left(u_{1}\right)^{-1} \gamma(u) . d\left(\gamma_{1}(u)\right)=d(\gamma(u))=u, \gamma_{1}\left(u_{1}\right)=$ $\gamma\left(u_{1}\right)^{-1} \gamma\left(u_{1}\right)=d\left(\gamma\left(u_{1}\right)\right)=u_{1}$, and $r\left(\gamma_{1}(u)\right)=d\left(\gamma\left(u_{1}\right)\right)=u_{1} \cdot \psi_{1}(x)=$ $\gamma_{1}(r(x)) x \gamma_{1}(d(x))^{-1}$ and inc: $G\left|\left\{u_{1}\right\} \rightarrow G\right| U_{2}$ effect a similarity of $\left(G \mid U_{2}, C\right)$ and $\left(G \mid\left\{u_{1}\right\}, \psi_{1 *}(C)\right)$, so $G \mid\left\{u_{1}\right\}$ is the Borel group of the same compact group that $G \mid\left\{u_{0}\right\}$ is. Thus we may as well assume $u_{0} \in U_{2}$.

Let $\mu$ be the Haar probability in $\psi_{*}(C)$ on $G \mid\left\{u_{0}\right\}$ and define $\nu^{u}$ by

$$
\int f(y) d \nu^{u}(y)=\int_{r^{-1}\left(\left\{u_{0}\right\}\right) \cap G \mid U_{2}}\left(\int_{G \mid\left\{u_{0}\right\}} f\left(\gamma(u)^{-1} z w\right) d \mu(x)\right) d \lambda^{u_{0}}(w)
$$

for $u \in U_{2} \cdot \nu^{u}$ is a probability measure; and by Fubini's Theorem, $u \mapsto$ $\int f(y) d \nu^{u}(y)$ is Borel. If $x \in G \mid U_{2}$ and $f \geqslant 0$, then 


$$
\begin{aligned}
\int f(x y) d \nu^{d(x)}(y) & =\iint f\left(x \gamma(d(x))^{-1} z w\right) d \mu(z) d \lambda^{u_{0}}(w) \\
= & \iint f\left(x \gamma(d(x))^{-1} \gamma(d(x)) x^{-1} \gamma(r(x))^{-1} z w\right) d \mu(z) d \lambda^{u_{0}}(w)
\end{aligned}
$$

since $\psi(x)^{-1}=\gamma(d(x)) x^{-1} \gamma(r(x))^{-1} \in G \mid\left\{u_{0}\right\}$ and $\mu$ is invariant. Hence

$$
\begin{aligned}
\int f(x y) d \nu^{d(x)}(y) & =\iint f\left(\gamma(r(x))^{-1} z w\right) d \mu(z) d \lambda^{u_{0}}(w) \\
& =\int f(y) d \nu^{r(x)}(y) .
\end{aligned}
$$

If $f \geqslant 0$ and $f$ is a $\lambda^{u}$-null Borel function, then

$$
\begin{array}{rlrl}
\int f(y) d \nu^{u}(y) & =\iint f\left(\gamma(u)^{-1} z w\right) d \mu(z) d \lambda^{u_{0}}(w) \\
& =\iint f\left(\gamma(u)^{-1} z w\right) d \lambda^{u_{0}}(w) d \mu(z) \\
& =0 & \text { by Tonelli's Theorem }
\end{array}
$$

because

$$
\begin{aligned}
\int f\left(\gamma(u)^{-1} z w\right) d \lambda^{u_{0}}(w) & =\int f\left(\left(\gamma(u)^{-1} z\right) w\right) d \lambda^{d\left(\gamma(u)^{-1} z\right)}(w) \\
& \sim \int f(w) d \lambda^{r\left(\gamma(u)^{-1} z\right)}(w)=\int f(w) d \lambda^{u}(w)=0 .
\end{aligned}
$$

If $f \geqslant 0$ and $f$ is $\nu^{u}$-null, then for a.a. $z, \int f\left(\gamma(u)^{-1} z w\right) d \lambda^{u_{0}}(w)=0$, and this implies $\int f(w) d \lambda^{u}(w)=0$. Thus $\nu^{u} \sim \lambda^{u}$.

Let $\nu$ be defined on $G \mid U_{2}$ by $\int f d \nu=\iint f(x) d \nu^{u}(x) d \tilde{\lambda}(u)$. Then $\nu$ is a probability and $(\nu, \tilde{\lambda})$ is a finite Haar measure. This proves $1 \Rightarrow 2$.

$(2 \Rightarrow 3)$ Let $\left(\nu_{1}, \mu\right)$ be a finite Haar measure with $r$-decomposition $\nu_{1}$ $=\int \nu^{u} d \mu(u)$ satisfying (3.12) on some i.r. Then by ergodicity $u \mapsto \nu^{u}(G)$ is essentially constant. This constant is finite so we may assume that it is 1 . Let $\lambda \in C$ be a symmetric probability. Define $\nu=\int \nu^{u} d \tilde{\lambda}$, so that $(\nu, \tilde{\lambda})$ is a finite Haar measure. Let $\lambda=\int \lambda^{u} d \tilde{\lambda}(u)$ be an $r$-decomposition of $\lambda, P=d \nu / d \lambda$. Using the decomposition $\nu=\int P \lambda^{u} d \tilde{\lambda}(u)$ and applying Corollary 3.14, one sees that the probability $\nu$ is as described by Theorem 3.9, so $\nu^{-1}$ is as in Theorem 4.2. Furthermore, if $\nu^{\prime}=(r, d)_{*}(\nu)$, then $\left(\nu^{\prime}, \tilde{\lambda}\right)$ is easily checked to be a Haar measure for $\mathcal{E}=(r, d)(G)$; and, as for $\nu^{-1},\left(\nu^{\prime}\right)^{-1}$ is as described by Corollary 4.3. Thus if we apply Theorem 4.4 to the finite measures $\nu^{-1}$ and $\left(\nu^{\prime}\right)^{-1}$, we see that the measures $\nu_{u v}$ obtained in that theorem must be $\lambda^{\prime}$-a.a. probabilities. Applying the translation formulae of Theorem 4.4, $\tilde{\lambda}$-a.a. $\nu_{u u}$ are probabilities.

Regarding $\mathcal{E}$ as an ergodic equivalence relation as in Example 2.7, there are measures $\beta_{u}$ on $[u]$ such that $\nu^{\prime}=\int \delta_{u} \times \beta_{u} d \tilde{\lambda}(u)$. Since $\left(\nu^{\prime}, \mu\right)$ is a Haar 
measure, there is a conull Borel set $U_{3} \subset U_{G}$ such that $(u, v) \in \mathcal{E} \mid U_{3} \Rightarrow \beta_{u}=$ $\beta_{v}$. By ergodicity, $u \rightarrow \beta_{u}\left(U_{G}\right)$ is a.e. a finite constant. Since $\mathscr{B}\left(U_{G}\right)$ is countably generated, the Carathéodory Extension Theorem implies that the set of probability measures on $U_{G}$ is countably separated. By ergodicity, then, $u \rightarrow \beta_{u}$ is essentially constant. Thus, there is essentially only one equivalence class $[u]$. This shows $\left(\mathcal{E},\left[v^{\prime}\right]\right)$ is essentially transitive, so $(G, C)$ is essentially transitive. This proves $2 \Rightarrow 3$.

$(3 \Rightarrow 4)$ Essential transitivity of $(G, C)$ implies essential transitivity of the associated principal groupoid $\left(\mathcal{E}, C^{\prime}\right)$. Applying Lemma 4.5 of [13] to $(r, d)_{*}(\lambda)$, on $\mathcal{E} \subset U_{G} \times U_{G}, \tilde{\lambda} \times \tilde{\lambda}=\int \delta_{u} \times \tilde{\lambda}(u) \in C ; \tilde{\lambda} \times \tilde{\lambda}$ is symmetric and $(\tilde{\lambda} \times \tilde{\lambda}, \tilde{\lambda})$ is a Haar measure. Referring to the decomposition of $\nu$ described by Theorem 4.4, for $u \in U_{0}, v_{u, u}$ are finite Haar measures for the the groups $G \mid\{u\}$, so the modular functions $\delta \mid(G \mid\{u\})$ must be identically 1 . Thus, the measures $\nu_{u u}$ are symmetric. Let $u$ belong to the conull equivalence class; let $\gamma$ be a Borel cross-section of $d$ on an i.r. $G \mid U_{1}$ of $G \mid U_{0}$ such that $\gamma(u)=u$ and $\gamma\left(U_{1}\right) \subset r^{-1}(\{u\})$, as at the beginning of the proof. Let $\eta_{u, u}=$ $v_{u, u} / \nu_{u, u}(G)$. Let $\eta_{v, w}=\gamma(v)^{-1} \cdot \eta_{u, u} \cdot \gamma(w)$; that is,

$$
\int f(y) d \eta_{v, w}=\int f\left(\gamma(v)^{-1} y \gamma(w)\right) v_{u, u}(G)^{-1} d v_{u, u}(y) .
$$

Then $\eta=\int \eta_{v, w} d \tilde{\lambda} \times \tilde{\lambda}(v, w) \in C$ and has $r$-decomposition $\eta=$ $\int\left(\int \eta_{v, w} d \tilde{\lambda}(w)\right) d \tilde{\lambda}(v) . \quad(\eta, \tilde{\lambda})$ is a Haar measure because

$$
\begin{aligned}
\int f(x y) & d \eta_{d(x), w}(y) d \tilde{\lambda}(w)=\int f\left(x \gamma(d(x))^{-1} y \gamma(w)\right) d \eta_{u, u}(y) d \tilde{\lambda}(w) \\
= & \int f\left(\gamma(r(x))^{-1} \gamma(r(x)) x \gamma(d(x))^{-1} y \gamma(w)\right) d \eta_{u, u}(y) d \tilde{\lambda}(w) \\
& =\int f\left(\gamma(r(x))^{-1} y \gamma(w)\right) d \eta_{u, u}(y) d \tilde{\lambda}(w)
\end{aligned}
$$

by invariance of the normalized Haar measure $\eta_{u, u}$

$$
=\int f(y) d \eta_{r(x), w}(y) d \tilde{\lambda}(w) .
$$

$\eta$ is symmetric because

$$
\begin{aligned}
\iint f\left(y^{-1}\right) & d \eta_{v, w}(y) d \tilde{\lambda} \times \tilde{\lambda}(v, w) \\
& =\iint f\left(\left(\gamma(v)^{-1} y \gamma(w)\right)^{-1}\right) d \eta_{u, u}(y) d \tilde{\lambda} \times \tilde{\lambda}(v, w) \\
& =\iint f\left(\gamma(w)^{-1} y^{-1} \gamma(v)\right) d \eta_{u, u}(y) d \tilde{\lambda} \times \tilde{\lambda}(v, w) \\
& =\iint f\left(\gamma(w)^{-1} y \gamma(v)\right) d \eta_{u, u}(y) d \tilde{\lambda} \times \tilde{\lambda}(w, v) \\
& =\iint f(y) d \eta_{v, w}(y) d \tilde{\lambda} \times \tilde{\lambda}(v, w) .
\end{aligned}
$$


Clearly $\eta(G)=1$, so we have proved $3 \Rightarrow 4$.

$(4 \Rightarrow 1)$ If $\lambda \in C$ is a symmetric probability such that $(\lambda, \tilde{\lambda})$ is a Haar measure, then, as we showed in $2 \Rightarrow 3, G$ is essentially transitive. Referring to the facts and notation described at the beginning of the proof, $\psi_{*}(C)$ contains $\psi_{*}(\lambda)$. Let $x \in G \mid\left\{u_{0}\right\}$.

$$
\int f(x y) d \psi_{*} \lambda(y)=\int f(x \psi(y)) d \lambda(y)=\int f(\psi(x \gamma(r(y)) y)) d \lambda(x),
$$

since

$$
\begin{aligned}
\psi(x \gamma(r(y)) y) & =\gamma(r(x)) x \gamma(r(y)) y \gamma(d(y))^{-1} \\
& =\gamma\left(u_{0}\right) x \psi(y)=x \psi(y) .
\end{aligned}
$$

For an $r$-decomposition $\lambda=\int \lambda^{u} d \tilde{\lambda}(u)$ and appropriate i.r. $G\left|U_{2} \subset G\right| U_{1}$, we have

$$
\begin{aligned}
\int f(\psi(x \gamma(r(y)) y)) d \lambda^{u}(y)=\int f \circ \psi(x \gamma(u) y) d \lambda^{d(x \gamma(u))}(y) \\
\quad=\int f \circ \psi(y) d \lambda^{r(x \gamma(u))}(y)=\int f \circ \psi(y) d \lambda^{u_{0}}(y) \\
=\int f \circ \psi(y) d \lambda^{r(\gamma(u))}(y) \\
=\int f \circ \psi(\gamma(u) y) d \lambda^{d(\gamma(u))}(y) \\
=\int f\left(\gamma(r(\gamma(u))) \gamma(u) y \gamma(d(y))^{-1}\right) d \lambda^{u}(y) \\
=\int f\left(\gamma(r(y)) y \gamma(d(y))^{-1}\right) d \lambda^{u}(y)=\int f(\psi(y)) d \lambda^{u}(y) .
\end{aligned}
$$

Thus $\int f(x y) d\left(\psi_{*} \lambda\right)(y)=\int f(y) d\left(\psi_{*} \lambda\right)(y)$. This proves that the 1.c. group to which $(G, C)$ is similar has a finite Haar measure, so is actually compact. Thus $4 \Rightarrow 1$.

COROLlaRY 5.2. An ergodic action by a compact group is essentially transitive.

Proof. Let $g$ be a compact group acting ergodically on the analytic probability space $(S, \mu)$ with $\mu$ quasi-invariant. Let $h$ be the Haar measure of g. Then by Example 3.16, $(\mu \times h, \mu)$ is a Haar measure for $(S \times \mathfrak{g},[\mu \times h])$. Since $h$ must be finite, $\mu \times h$ is finite. By the theorem, $(S \times g, \mu \times h)$ is essentially transitive. This implies that there is a conull orbit in $S$, so the action is essentially transitive.

The proof afforded by Theorem 5.1 of this well-known result is entirely of a measure-theoretic character. 


\section{REFERENCES}

1. L. Auslander and C. C. Moore, Unitary representations of solvable Lie groups, Mem. Amer. Math. Soc. No. 62 (1966).

2. J. Dixmier, Algèbres quasi-unitaires, Comment. Math. Helv. 26 (1952), 275-322.

3. E. Effros, Global structure in von Neumann algebras, Trans. Amer. Math. Soc. 121 (1966), 434-454.

4. J. Glimm, Families of induced representations, Pacific J. Math. 12 (1962), 885-911.

5. P. Hahn, Haar measure and convolution algebras on ergodic groupoids, Ph.D. Thesis, Harvard Univ., Cambridge, Mass., 1975.

6. _ The regular representations of measure groupoids, Trans. Amer. Math. Soc. 242 (1978), 35-72.

7. W. Kreiger, On constructing non-*-isomorphic hyperfinite factors of type III, J. Functional Analysis 6 (1970), 97-109.

8. C. Kuratowski, Topologie, Vol. I, 4th ed., PWN, Warsaw, 1958.

9. G. W. Mackey, Borel structures in groups and their duals, Trans. Amer. Math. Soc. 85 (1957), 265-311.

10. , Ergodic theory, group theory, and differential geometry, Proc. Nat. Acad. Sci. U.S.A. 50 (1963), 1184-1191.

11. Ergodic theory and virtual groups, Math. Ann. 166 (1966), 187-207.

12. F. J. Murray and J. von Neumann, On rings of operators, Ann. of Math. (2) 37 (1936), 116-229.

13. A. Ramsay, Virtual groups and group actions, Advances in Math. 6 (1971), 253-322.

14. Boolean duals of virtual groups, J. Functional Analysis 15 (1974), 56-101.

15. M. Samuelides and J.-L. Sauvageot, Algèbre de Krieger d'un système dynamique, C. R. Acad. Sci. Paris Sér. A-B 280 (1975), A709-A712.

16. A. K. Seda, Un concept de mesures invariantes pour les groupoïdes topologiques, C. R. Acad. Sci. Paris Sér. A-B 280 (1975), A1603-A1605.

17. J. J. Westman, Harmonic analysis on groupoids, Pacific J. Math. 27 (1968), 621-632.

18. __ Nontransitive groupoid algebras (unpublished).

19. __ Ergodic groupoid algebras and their representations (unpublished).

Department of Mathematics, University of California, Berkeley, California 94720

Current address: 10 Soldiers Field Park 10C, Boston, Massachusetts 02163 